\title{
A Galerkin FEM for Riesz space-fractional CNLS
}

\author{
Xiaogang Zhu' ${ }^{*}$, Yufeng $\mathrm{Nie}^{2}$, Zhanbin Yuan², Jungang Wang ${ }^{2}$ and Zongze Yang ${ }^{2}$
}

"Correspondence: zhuxg590@yeah.net

'School of Science, Shaoyang University, Shaoyang, P.R. China Full list of author information is available at the end of the article

\begin{abstract}
In quantum physics, fractional Schrödinger equation is of particular interest in the research of particles on stochastic fields modeled by the Lévy processes, which was derived by extending the Feynman path integral over the Brownian paths to a path integral over the trajectories of Lévy fights. In this work, a fully discrete finite element method (FEM) is developed for the Riesz space-fractional coupled nonlinear Schrödinger equations (CNLS), conjectured with a linearized Crank-Nicolson discretization. The error estimate and mass conservative property are discussed. It is showed that the proposed method is decoupled and convergent with optimal orders in $L^{2}$-sense. Numerical examples are performed to support our theoretical results.
\end{abstract}

MSC: 35R11;65M60;65M12

Keywords: Space-fractional CNLS; FEM; Convergent analysis

\section{Introduction}

An explosive interest has been gained to the fractional calculus and its applications during recent years. It has been known as an extension of classical calculus to non-integer orders, but compares favorably with classical calculus in modeling various applications, including image processing [1], coupled pendulums [2], capacitor microphone [3], optimal control [4], and anomalous diffusion [5, 6].

As a superior tool to describe the anomalous dispersion in the complex heterogeneous aquifer and the medium with fractal geometry $[7,8]$, fractional partial differential equations (PDEs) have been used to describe many problems in science and engineering. Their theoretical properties were extensively investigated $[9,10]$, and their potential applications in various subjects have nurtured numerous robust methods designed for solving them numerically, including spectral method [11], finite difference methods (FDMs) [12-16], FEMs [17-20], variational iteration methods [21, 22], and differential quadrature methods [23,24]. The main difficulty of numerical methods lies in approximating the fractional derivatives. There exist several ways to discretize fractional derivatives, such as $L^{1}$-formula [25], Grünwald-Letnikov operator [26], and other fractional differences [27-29].

In this paper, regarding the current interest in fractional PDEs as effective models, we showcase an efficient FEM for the Riesz space-fractional CNLS

$$
\mathrm{i} \partial_{t} u+\gamma \partial_{x}^{2 \alpha} u+\lambda\left(|u|^{2}+\rho|v|^{2}\right) u=0
$$

(c) The Author(s) 2019. This article is distributed under the terms of the Creative Commons Attribution 4.0 International License (http://creativecommons.org/licenses/by/4.0/), which permits unrestricted use, distribution, and reproduction in any medium, provided you give appropriate credit to the original author(s) and the source, provide a link to the Creative Commons license, and indicate if changes were made. 


$$
\mathrm{i} \partial_{t} v+\gamma \partial_{x}^{2 \alpha} v+\lambda\left(\rho|u|^{2}+|v|^{2}\right) v=0, \quad t \in(0, T]
$$

with $\mathrm{i}^{2}=-1$, real parameters $\gamma, \lambda, \rho, 1 / 2<\alpha \leq 1$, and the Riesz derivative

$$
\begin{aligned}
& \partial_{x}^{2 \alpha} u(x, t)=\frac{-1}{2 \cos (\alpha \pi)}\left\{D_{L}^{2 \alpha} u(x, t)+D_{R}^{2 \alpha} u(x, t)\right\}, \\
& D_{L}^{2 \alpha} u(x, t)=\frac{1}{\Gamma(2-2 \alpha)} \frac{\partial^{2}}{\partial x^{2}} \int_{-\infty}^{x}(x-\xi)^{1-2 \alpha} u(\xi, t) d \xi, \\
& D_{R}^{2 \alpha} u(x, t)=\frac{1}{\Gamma(2-2 \alpha)} \frac{\partial^{2}}{\partial x^{2}} \int_{x}^{+\infty}(\xi-x)^{1-2 \alpha} u(\xi, t) d \xi,
\end{aligned}
$$

where $\Gamma(\cdot)$ denotes Euler's Gamma function. $\partial_{x}^{2 \alpha} v$ can be analogously defined as $\partial_{x}^{2 \alpha} u$. Since the true solutions to Eqs. (1.1)-(1.2) subjected to suitable initial values will polynomially $(\alpha \neq 1)$ or exponentially $(\alpha=1)$ decay to zero when $|x| \rightarrow \infty$ [30], we consider the system truncated on a bounded domain $\Omega=(a, b)$ and take the initial and boundary conditions as follows:

$$
\begin{aligned}
& u(x, 0)=u_{0}(x), \quad v(x, 0)=v_{0}(x), \quad x \in \Omega \cup \partial \Omega, \\
& u(x, t)=0, \quad v(x, t)=0, \quad(x, t) \in \partial \Omega \times(0, T],
\end{aligned}
$$

where $\partial \Omega$ is its boundary, $a \ll 0, b \gg 0$, and $u_{0}(x), v_{0}(x)$ are prescribed initial functions. In particular, it is noted that Eqs. (1.1)-(1.2) recover to the classical CNLS when $\alpha=1$, which was raised by Benney and Newell for two interacting wave trains in a weakly nonlinear conservative system [31].

The single space-fractional nonlinear Schrödinger equation (NLS) was first generated by Laskin [32, 33], via extending the Feynman path integral over the Brownian paths to a new path integral over the Lévy quantum mechanical paths. Some important physical properties of self-similarity of the quantum mechanical path can be captured by this new approach. Besides, the fractional derivatives in NLS/CNLS can modify the shape of wave, while the nonlinearity and dispersion effects remain unchanged. Therefore, the spacefractional NLS/CNLS have been the topics of intense research recently. The existence and uniqueness of their global smooth solutions were investigated by [34-36]. The local wellposedness and ill-posedness in Sobolev spaces for power-type nonlinearities were studied in [37]. Moreover, there have been numerous works devoted to exploring the theoretical properties for those equations of different types; we refer the readers to [30, 38-41] for overall views. In the aspect of numerical algorithms for the space-fractional NLS/CNLS, Herzallah and Gepreel proposed an Adomian decomposition method to construct an approximate solution for the time-space-fractional NLS with cubic nonlinearity [42]. Atangana addressed the variable-order NLS of nonlocal type solved by the Crank-Nicolson method [43]. Amore et al. suggested a collocation approach based on little Sinc functions for the space-fractional NLS on a uniform grid [44], where good convergent behaviors are observed. In [45], the authors presented a second-order FDM for the Riesz spacefractional NLS and proved that it can conserve the discrete mass and energy. Klein et al. provided a detailed numerical study for the nonlocal NLS by Fourier spectral method [46]. Wang et al. established two mass conservative FDMs for the Riesz space-fractional CNLS $[47,48]$, whose stability and convergence were discussed. Liu et al. solved the fractional 
NLS by the implicit and explicit-implicit FDMs, and the fractional CNLS by an implicit FDM [49]. Recently, a linearized compact alternating direction implicit method was constructed for the space-fractional NLS [50], by using a novel $\alpha$-dependent fourth-order fractional compact difference operator, which turns out to be stable and convergent with second-order in time and fourth-order in space. The Legendre spectral and pseudospectral methods can be found in $[51,52]$. Zhu et al. developed an implicit FEM for spacefractional NLS [53]. Li et al. proposed a fast linearized conservative FEM for the strongly coupled space-fractional NLS [54], which uses Krylov subspace solvers with circulant preconditioners to handle the resulting Toeplitz-like systems.

Unlike the classical NLS, it is quite difficult to solve the fractional NLS analytically, let alone the space-fractional CNLS. Hence, the numerical methods are especially important. However, the existing methods are almost limited to FDM, only a few works on FEM have been done for the space-fractional NLS/CNLS. It is well known that FDMs are not suitable for complex regional problems and have a higher regularity requirement to guarantee their convergence in fractional cases, so do the spectral methods. For example, the scheme in [47] using fractional centered difference to deal with Riesz derivative requires $u, v \in C^{5}(\mathbb{R})$. As a common method, FEM compares favorably with FDM. It is widely used in the continuous medium and field problems on complex domains in engineering, and achieves higher accuracy with lower regularity requirements. The numerical simulation of the space-fractional NLS/CNLS by FEM helps to know the shape and long time behavior of the solitons governed by the fractional derivatives and their changes with different $\alpha$. The main contribution of this article is to propose a fully discrete explicit-implicit FEM to solve the Riesz space-fractional CNLS on bounded domains, which is decoupled. We prove that it is conservative to mass and convergent with optimal orders in $L^{2}$-norm. The proposed method is applied to simulate the propagation of solitons, and some meaningful physical results are found. Since the scheme is linearized, an extra iterative loop is avoided, which is beneficial to improving computing efficiency. The novelty lies in the construction of a decoupled and mass conservative FEM by decomposing $u, v$ in Eqs. (1.1)-(1.2) into their real and imaginary parts, which can reach high accuracy with low computing burden and regularity.

The outline is organized as follows. In Sect. 2, we give some auxiliary results related to the fractional calculus, which will be used throughout the paper. Section 3 studies the weak problem along with nonlocal bilinear form. In Sect. 4, we construct the discrete scheme with FEM to discretize Eqs. (1.1)-(1.4) and prove that it is stable. In Sect. 5, the convergence is derived, and to evaluate the effectiveness of our method, numerical tests are covered in Sect. 6.

\section{Auxiliary results}

In the sequel, we employ

$$
\|u\|_{L^{2}(\mathbb{R})}=(u, u)_{L^{2}(\mathbb{R})}^{1 / 2}, \quad(u, v)_{L^{2}(\mathbb{R})}=\int_{\mathbb{R}} u v d x .
$$

Hereafter, $(\cdot, \cdot)$ will be consistently used to stand for the above inner product. In view of $[18,19]$, for $\mu>0$, the left, right, and symmetric fractional semi-norms and norms are defined as

$$
|u|_{J_{L}^{\mu}(\mathbb{R})}=\left\|D_{L}^{\mu} u\right\|_{L^{2}(\mathbb{R})}, \quad\|u\|_{J^{\mu}(\mathbb{R})}=\left\{\|u\|_{L^{2}(\mathbb{R})}^{2}+|u|_{J_{L}^{\mu}(\mathbb{R})}^{2}\right\}^{1 / 2},
$$




$$
\begin{aligned}
& |u|_{J_{R}^{\mu}(\mathbb{R})}=\left\|D_{R}^{\mu} u\right\|_{L^{2}(\mathbb{R})}, \quad\|u\|_{J_{R}^{\mu}(\mathbb{R})}=\left\{\|u\|_{L^{2}(\mathbb{R})}^{2}+|u|_{J_{R}^{\mu}(\mathbb{R})}^{2}\right\}^{1 / 2}, \\
& |u|_{J_{S}^{\mu}(\mathbb{R})}=\left|\left(D_{L}^{\mu} u, D_{R}^{\mu} u\right)+\left(D_{R}^{\mu} u, D_{L}^{\mu} u\right)\right|^{1 / 2}, \quad\|u\|_{J_{S}^{\mu}(\mathbb{R})}=\left\{\|u\|_{L^{2}(\mathbb{R})}^{2}+|u|_{J_{S}^{\mu}(\mathbb{R})}^{2}\right\}^{1 / 2},
\end{aligned}
$$

and $J_{L}^{\mu}(\mathbb{R}), J_{R}^{\mu}(\mathbb{R}), J_{S}^{\mu}(\mathbb{R})$ are denoted as the closures of $C^{\infty}(\mathbb{R})$ with regard to $\|\cdot\|_{J^{\mu}}^{\mu}(\mathbb{R})$, $\|\cdot\|_{J_{R}^{\mu}(\mathbb{R})}$, and $J_{S}^{\mu}(\mathbb{R})$, respectively. Also, let $\mathcal{F}[u]$ be the Fourier transform of a prescribed function $u(x) \in L^{1}(\mathbb{R})$, with variable $\omega$, i.e., $\mathcal{F}[u]=\int_{\mathbb{R}} u(x) e^{-i \omega x} d x$. Then, the fractional Sobolev semi-norm and norm are defined by

$$
|u|_{H^{\mu}(\mathbb{R})}=\left\||\omega|^{\mu} \mathcal{F}[u]\right\|_{L^{2}\left(\mathbb{R}_{\omega}\right)}, \quad\|u\|_{H^{\mu}(\mathbb{R})}=\left\{\|u\|_{L^{2}(\mathbb{R})}^{2}+|u|_{H^{\mu}(\mathbb{R})}^{2}\right\}^{1 / 2},
$$

and $H^{\mu}(\mathbb{R})$ is denoted as the closure of $C^{\infty}(\mathbb{R})$ with regard to $\|\cdot\|_{H^{\mu}(\mathbb{R})}$.

Lemma 2.1 For $\mu>0$, we have the identity in $L^{2}$-sense

$$
\left(D_{L}^{\mu} u, D_{R}^{\mu} u\right)+\left(D_{R}^{\mu} u, D_{L}^{\mu} u\right)=2 \cos (\mu \pi)\left\|D_{L}^{\mu} u\right\|_{L^{2}(\mathbb{R})}^{2} .
$$

Proof Let $\hat{u}(\omega)=\mathcal{F}[u]$. Then based on [26], there exist

$$
\mathcal{F}\left[D_{L}^{\mu} u\right]=(\mathrm{i} \omega)^{\mu} \hat{u}(\omega), \quad \mathcal{F}\left[D_{R}^{\mu} u\right]=(-\mathrm{i} \omega)^{\mu} \hat{u}(\omega) .
$$

Observing that

$$
\overline{(i \omega)^{\mu}}= \begin{cases}\exp (-\mathrm{i} \mu \pi) \overline{(-\mathrm{i} \omega)^{\mu}} & \text { for } \omega \geq 0 \\ \exp (\mathrm{i} \mu \pi) \overline{(-\mathrm{i} \omega)^{\mu}} & \text { for } \omega<0\end{cases}
$$

and via Plancherel's theorem, we immediately have

$$
\begin{aligned}
\left(D_{L}^{\mu} u, D_{R}^{\mu} u\right)= & \left((\mathrm{i} \omega)^{\mu} \hat{u},(-\mathrm{i} \omega)^{\mu} \hat{u}\right) \\
= & \int_{-\infty}^{0}(\mathrm{i} \omega)^{\mu} \hat{u} \overline{(-\mathrm{i} \omega)^{\mu} \hat{u}} d \omega+\int_{0}^{\infty}(\mathrm{i} \omega)^{\mu} \hat{u} \overline{(-\mathrm{i} \omega)^{\mu} \hat{u}} d \omega \\
= & \int_{-\infty}^{0}(\mathrm{i} \omega)^{\mu} \hat{u} \exp (-\mathrm{i} \mu \pi) \overline{(\mathrm{i} \omega)^{\mu} \hat{u}} d \omega \\
& +\int_{0}^{\infty}(\mathrm{i} \omega)^{\mu} \hat{u} \exp (\mathrm{i} \mu \pi) \overline{(\mathrm{i} \omega)^{\mu} \hat{u}} d \omega \\
= & \cos (\mu \pi) \int_{-\infty}^{\infty}(\mathrm{i} \omega)^{\mu} \hat{u} \overline{(\mathrm{i} \omega)^{\mu} \hat{u}} d \omega \\
& +\mathrm{i} \sin (\mu \pi)\left(\int_{0}^{\infty}\left|(\mathrm{i} \omega)^{\mu} \hat{u}\right|^{2} d \omega-\int_{-\infty}^{0}\left|(\mathrm{i} \omega)^{\mu} \hat{u}\right|^{2} d \omega\right) .
\end{aligned}
$$

In the same fashion, we obtain

$$
\begin{aligned}
& \left(D_{R}^{\mu} u, D_{L}^{\mu} u\right)=\left((-\mathrm{i} \omega)^{\mu} \hat{u},(\mathrm{i} \omega)^{\mu} \hat{u}\right) \\
& =\int_{-\infty}^{0}(-\mathrm{i} \omega)^{\mu} \hat{u} \overline{(\mathrm{i} \omega)^{\mu} \hat{u}} d \omega+\int_{0}^{\infty}(-\mathrm{i} \omega)^{\mu} \hat{u} \overline{(\mathrm{i} \omega) \hat{u}} d \omega
\end{aligned}
$$




$$
\begin{aligned}
= & \int_{-\infty}^{0}(-\mathrm{i} \omega)^{\mu} \hat{u} \exp (\mathrm{i} \mu \pi) \overline{(-\mathrm{i} \omega)^{\mu} \hat{u}} d \omega \\
& +\int_{0}^{\infty}(-\mathrm{i} \omega)^{\mu} \hat{u} \exp (-\mathrm{i} \mu \pi) \overline{(-\mathrm{i} \omega)^{\mu} \hat{u}} d \omega \\
= & \cos (\mu \pi) \int_{-\infty}^{\infty}(-\mathrm{i} \omega)^{\mu} \hat{u} \overline{(-\mathrm{i} \omega)^{\mu} \hat{u}} d \omega \\
& +\mathrm{i} \sin (\mu \pi)\left(\int_{-\infty}^{0}\left|(-\mathrm{i} \omega)^{\mu} \hat{u}\right|^{2} d \omega-\int_{0}^{\infty}\left|(-\mathrm{i} \omega)^{\mu} \hat{u}\right|^{2} d \omega\right) .
\end{aligned}
$$

On the other hand, there holds $\left\|D_{L}^{\mu} u\right\|_{L^{2}(\mathbb{R})}^{2}=\int_{-\infty}^{\infty}|\omega|^{2 \mu}|\hat{u}|^{2} d \omega$. Hence Eq. (2.1) is straightforward by the sum of Eqs. (2.2)-(2.3). The proof is completed.

Lemma $2.2([18])$ For $\mu>0, \mu \neq n-1 / 2, n \in \mathbb{N}, J_{L}^{\mu}(\mathbb{R}), J_{R}^{\mu}(\mathbb{R}), J_{S}^{\mu}(\mathbb{R})$, and $H^{\mu}(\mathbb{R})$ are equal with their equivalent semi-norms and norms.

Let $\Omega \subset \mathbb{R}, \mu \neq n-1 / 2, n \in \mathbb{N}$, and $J_{L, 0}^{\mu}(\Omega), J_{R, 0}^{\mu}(\Omega), J_{S, 0}^{\mu}(\Omega)$, and $H_{0}^{\mu}(\Omega)$ be the closures of $C_{0}^{\infty}(\Omega)$ with regard to $\|\cdot\|_{J_{L}^{\mu}(\Omega)},\|\cdot\|_{J_{R}^{\mu}(\Omega)},\|\cdot\|_{J_{S}^{\mu}(\Omega)}$, and $\|\cdot\|_{H^{\mu}(\Omega)}$, respectively. Then the following lemmas are admitted.

Lemma $2.3([18])$ For $\mu>0, \mu \neq n-1 / 2, n \in \mathbb{N}, J_{L, 0}^{\mu}(\Omega), J_{R, 0}^{\mu}(\Omega), J_{S, 0}^{\mu}(\Omega)$, and $H_{0}^{\mu}(\Omega)$ are equal with their equivalent semi-norms and norms.

Lemma 2.4 (Fractional Poincaré-Friedrichs [18]) For $\mu>0, u \in J_{L, 0}^{\mu}(\Omega)$, and $0<s<\mu$, one has

$$
\|u\|_{L^{2}(\Omega)} \leq C|u|_{J_{L, 0}^{\mu}(\Omega)}, \quad|u|_{J_{L, 0}^{s}(\Omega)} \leq C|u|_{J_{L, 0}^{\mu}(\Omega)},
$$

and for $u \in J_{R, 0}^{\mu}(\Omega), 0<s<\mu$, one has

$$
\|u\|_{L^{2}(\Omega)} \leq C|u|_{J_{R, 0}^{\mu}(\Omega)}, \quad|u|_{J_{R, 0}^{s}(\Omega)} \leq C|u|_{J_{R, 0}^{\mu}(\Omega)}
$$

If $u \in H_{0}^{\mu}(\Omega), 0<s<\mu, \mu \neq n-1 / 2, n \in \mathbb{N}$, the analogous result is obtained.

In what follows, for ease of exposition, we adopt $\|\cdot\|_{0, \Omega}=\|\cdot\|_{L^{2}(\Omega)}$, $\|\cdot\|_{\infty, \Omega}=$ ess $\sup _{x \in \Omega}|\cdot|$, and $\|\cdot\|_{p, \Omega}=\|\cdot\|_{H^{p}(\Omega)}, p \in \mathbb{R}$. " $a \lesssim b$ ", " $a \gtrsim b$ " mean " $a \leq C b$ " and " $a \geq$ $C b$ ", respectively; $C$ is a general constant which varies at different occasions. Moreover,

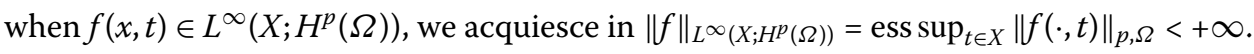
$f(x, t) \in L^{\infty}\left(0, T ; H^{p}(\Omega)\right)$ will be abbreviated to $f(x, t) \in L^{\infty}\left(H^{p}\right)$, if no ambiguity is possible.

\section{Weak problem}

Define the semi-norm and energy norm as follows:

$$
|u|_{E}=\left|\left(D_{L}^{\alpha} u, D_{R}^{\alpha} u\right)+\left(D_{R}^{\alpha} u, D_{L}^{\alpha} u\right)\right|^{1 / 2}, \quad\|u\|_{E}=\left\{\|u\|_{0, \Omega}^{2}+|u|_{E}^{2}\right\}^{1 / 2}
$$

and decompose $u(x), v(x)$ into their real and imaginary parts by

$$
u(x)=u_{1}(x)+\mathrm{i} u_{2}(x), \quad v(x)=v_{1}(x)+\mathrm{i} v_{2}(x),
$$


then Eqs. (1.1)-(1.2) can be recast as the coupled system

$$
\begin{aligned}
& \partial_{t} u_{1}+\gamma \partial_{x}^{2 \alpha} u_{2}+\lambda\left(\left|u_{1}\right|^{2}+\left|u_{2}\right|^{2}+\rho\left|v_{1}\right|^{2}+\rho\left|v_{2}\right|^{2}\right) u_{2}=0, \\
& \partial_{t} u_{2}-\gamma \partial_{x}^{2 \alpha} u_{1}-\lambda\left(\left|u_{1}\right|^{2}+\left|u_{2}\right|^{2}+\rho\left|v_{1}\right|^{2}+\rho\left|v_{2}\right|^{2}\right) u_{1}=0, \\
& \partial_{t} v_{1}+\gamma \partial_{x}^{2 \alpha} v_{2}+\lambda\left(\rho\left|u_{1}\right|^{2}+\rho\left|u_{2}\right|^{2}+\left|v_{1}\right|^{2}+\left|v_{2}\right|^{2}\right) v_{2}=0, \\
& \partial_{t} v_{2}-\gamma \partial_{x}^{2 \alpha} v_{1}-\lambda\left(\rho\left|u_{1}\right|^{2}+\rho\left|u_{2}\right|^{2}+\left|v_{1}\right|^{2}+\left|v_{2}\right|^{2}\right) v_{1}=0, \quad t \in(0, T] .
\end{aligned}
$$

By using Lemma 2.1, we give the weak problem: seek $u_{1}, u_{2}, v_{1}, v_{2} \in L^{2}\left(0, T ; H_{0}^{\alpha}(\Omega)\right) \cap$ $C^{0}\left(0, T ; L^{2}(\Omega)\right)$, for any $\chi_{u}^{1}, \chi_{u}^{2}, \chi_{v}^{1}, \chi_{v}^{2} \in H_{0}^{\alpha}(\Omega)$, such that

$$
\begin{aligned}
& \left(\partial_{t} u_{1}, \chi_{u}^{1}\right)-\gamma \Lambda\left(u_{2}, \chi_{u}^{1}\right)+\lambda\left(\left(\left|u_{1}\right|^{2}+\left|u_{2}\right|^{2}+\rho\left|v_{1}\right|^{2}+\rho\left|v_{2}\right|^{2}\right) u_{2}, \chi_{u}^{1}\right)=0, \\
& \left(\partial_{t} u_{2}, \chi_{u}^{2}\right)+\gamma \Lambda\left(u_{1}, \chi_{u}^{2}\right)-\lambda\left(\left(\left|u_{1}\right|^{2}+\left|u_{2}\right|^{2}+\rho\left|v_{1}\right|^{2}+\rho\left|v_{2}\right|^{2}\right) u_{1}, \chi_{u}^{2}\right)=0, \\
& \left(\partial_{t} v_{1}, \chi_{v}^{1}\right)-\gamma \Lambda\left(v_{2}, \chi_{v}^{1}\right)+\lambda\left(\left(\rho\left|u_{1}\right|^{2}+\rho\left|u_{2}\right|^{2}+\left|v_{1}\right|^{2}+\left|v_{2}\right|^{2}\right) v_{2}, \chi_{v}^{1}\right)=0, \\
& \left(\partial_{t} v_{2}, \chi_{v}^{2}\right)+\gamma \Lambda\left(v_{1}, \chi_{v}^{2}\right)-\lambda\left(\left(\rho\left|u_{1}\right|^{2}+\rho\left|u_{2}\right|^{2}+\left|v_{1}\right|^{2}+\left|v_{2}\right|^{2}\right) v_{1}, \chi_{v}^{2}\right)=0, \\
& u_{1}(x, 0)=u_{1}^{0}(x), \quad u_{2}(x, 0)=u_{2}^{0}(x), \\
& v_{1}(x, 0)=v_{1}^{0}(x), \quad v_{2}(x, 0)=v_{2}^{0}(x),
\end{aligned}
$$

subjected to zero boundary values, where

$$
\Lambda(u, v)=\frac{1}{2 \cos (\alpha \pi)}\left(D_{L}^{\alpha} u, D_{R}^{\alpha} v\right)+\frac{1}{2 \cos (\alpha \pi)}\left(D_{R}^{\alpha} u, D_{L}^{\alpha} v\right),
$$

and $u_{0}^{1}(x), u_{0}^{2}(x), v_{0}^{1}(x), v_{0}^{2}(x)$ are prescribed initial functions.

Theorem 3.1 The bilinear form $\Lambda(\cdot, \cdot)$ satisfies

$$
\begin{aligned}
& \Lambda(u, v) \lesssim\|u\|_{E}\|v\|_{E}, \\
& \Lambda(u, u) \gtrsim\|u\|_{E}^{2},
\end{aligned}
$$

and the symmetry property.

Proof Due to Hölder's inequality, we have

$$
\Lambda(u, v) \leq 1 \frac{1}{2 \cos (\alpha \pi)}\left\|D_{L}^{\alpha} u\right\|_{0, \Omega}\left\|D_{R}^{\alpha} v\right\|_{0, \Omega}+\left|\frac{1}{2 \cos (\alpha \pi)}\right|\left\|D_{R}^{\alpha} u\right\|_{0, \Omega}\left\|D_{L}^{\alpha} v\right\|_{0, \Omega} .
$$

Considering the model only on $\Omega$, for $u, v \in H_{0}^{\alpha}(\Omega)$, we denote their extensions to $\mathbb{R}$ by zero beyond $\Omega$ to be $\tilde{u}, \tilde{v}$, respectively. Then, by the inequality

$$
\forall a, b, c, d>0, \quad a b+c d \leq \sqrt{a^{2}+c^{2}} \sqrt{b^{2}+d^{2}},
$$

we obtain

$$
\Lambda(u, v) \leq C_{s} \sqrt{\left\|D_{L}^{\alpha} \tilde{u}\right\|_{L^{2}(\mathbb{R})}^{2}+\left\|D_{R}^{\alpha} \tilde{u}\right\|_{L^{2}(\mathbb{R})}^{2}} \sqrt{\left\|D_{L}^{\alpha} \tilde{v}\right\|_{L^{2}(\mathbb{R})}^{2}+\left\|D_{R}^{\alpha} \tilde{v}\right\|_{L^{2}(\mathbb{R})}^{2}},
$$


where

$$
C_{s}=C_{\alpha} / 2, \quad C_{\alpha}=\left|\frac{1}{\cos (\alpha \pi)}\right| .
$$

Further, for Plancherel's theorem, one gets

$$
\left\|D_{L}^{\alpha} \tilde{u}\right\|_{L^{2}(\mathbb{R})}^{2}=\left\||\mathrm{i} \omega|^{\alpha} \mathcal{F}[\tilde{u}]\right\|_{L^{2}\left(\mathbb{R}_{\omega}\right)}^{2}=\left\||-\mathrm{i} \omega|^{\alpha} \mathcal{F}[\tilde{u}]\right\|_{L^{2}\left(\mathbb{R}_{\omega}\right)}^{2}=\left\|D_{R}^{\alpha} \tilde{u}\right\|_{L^{2}(\mathbb{R})}^{2},
$$

which yields

$$
\Lambda(u, v) \leq C_{\alpha}\left\|D_{L}^{\alpha} \tilde{u}\right\|_{L^{2}(\mathbb{R})}\left\|D_{L}^{\alpha} \tilde{v}\right\|_{L^{2}(\mathbb{R})} .
$$

From Lemma 2.1 and $\operatorname{Supp}\left(D_{L}^{\alpha} \tilde{u} \overline{D_{R}^{\alpha} \tilde{u}}+D_{R}^{\alpha} \tilde{u} \overline{D_{L}^{\alpha} \tilde{u}}\right) \subseteq \Omega$, it follows that

$$
\Lambda(u, v) \leq C_{\alpha} C_{s}|u|_{E}|v|_{E} \lesssim\|u\|_{E}\|v\|_{E} .
$$

As for the coercivity, clearly, by Lemma 2.1, there holds

$$
\Lambda(u, u)=\left|\frac{1}{2 \cos (\alpha \pi)}\right|\left|\left(D_{L}^{\alpha} u, D_{R}^{\alpha} u\right)+\left(D_{R}^{\alpha} u, D_{L}^{\alpha} u\right)\right| \gtrsim|u|_{E}^{2} .
$$

Therefore, using Lemma 2.3 and the fractional Poincaré-Friedrichs inequalities, $\Lambda(u, u) \gtrsim$ $\|u\|_{E}^{2}$ is an immediate consequence, which ends its proof.

\section{A fully discrete FEM}

In this section, we propose a fully discrete FEM to approximate the solutions of Eqs. (1.1)(1.4). Let $\tau=T / N, t_{j}=j \tau, j=0,1, \ldots, N$, and $N \in \mathbb{Z}^{+}$. For a sequence of functions $\left\{f^{n}\right\}_{n=1}^{N}$ in the temporal direction, we introduce

$$
\bar{\partial}_{t} f^{n}=\frac{f^{n}-f^{n-1}}{\tau}, \quad \bar{f}^{n-\frac{1}{2}}=\frac{f^{n}+f^{n-1}}{2}, \quad \hat{f}^{n-\frac{1}{2}}=\frac{3 f^{n-1}-f^{n-2}}{2} .
$$

To perform FEM, a spatial mesh $\Omega_{h}$ is given, with $h=(b-a) / M$, i.e.,

$$
a=x_{0}<x_{1}<\cdots<x_{M}=b, \quad M \in \mathbb{Z}^{+},
$$

for $\Omega$. Then the finite element subspace $\mathcal{V}_{h} \subset H_{0}^{\alpha}(\Omega)$ is defined as

$$
\mathcal{V}_{h}=\left\{v:\left.v\right|_{\Omega_{j}} \in \Pi_{k-1}, \Omega_{j}=\left[x_{j-1}, x_{j}\right], j=1,2, \ldots, M\right\},
$$

where $\Pi_{k-1}$ is a set of polynomials of degree at most $k-1$ on $\Omega_{j}$.

To avoid iterative loop, we discretize Eqs. (1.1)-(1.4) by a linearized Crank-Nicolson scheme, where a standard extrapolation method is used to treat the nonlinear terms. An explicit-implicit FEM is derived, which reads: seek $u_{1, h}^{n}, u_{2, h}^{n}, v_{1, h}^{n}, v_{2, h}^{n} \in \mathcal{V}_{h}, n=1,2, \ldots, N$, for any $\chi_{1, h}^{1}, \chi_{2, h}^{1}, \chi_{1, h}^{2}, \chi_{2, h}^{2} \in \mathcal{V}_{h}$, to satisfy

$$
\left(\bar{\partial}_{t} u_{1, h}^{n}, \chi_{u, h}^{1}\right)-\gamma \Lambda\left(\bar{u}_{2, h}^{n-\frac{1}{2}}, \chi_{u, h}^{1}\right)+\lambda\left(\hat{G}_{u}^{n-\frac{1}{2}} \bar{u}_{2, h}^{n-\frac{1}{2}}, \chi_{u, h}^{1}\right)=0,
$$




$$
\begin{aligned}
& \left(\bar{\partial}_{t} u_{2, h}^{n}, \chi_{u, h}^{2}\right)+\gamma \Lambda\left(\bar{u}_{1, h}^{n-\frac{1}{2}}, \chi_{u, h}^{2}\right)-\lambda\left(\hat{G}_{u}^{n-\frac{1}{2}} \bar{u}_{1, h}^{n-\frac{1}{2}}, \chi_{u, h}^{2}\right)=0, \\
& \left(\bar{\partial}_{t} v_{1, h}^{n}, \chi_{v, h}^{1}\right)-\gamma \Lambda\left(\bar{v}_{2, h}^{n-\frac{1}{2}}, \chi_{v, h}^{1}\right)+\lambda\left(\hat{G}_{v}^{n-\frac{1}{2}} \bar{v}_{2, h}^{n-\frac{1}{2}}, \chi_{v, h}^{1}\right)=0, \\
& \left(\bar{\partial}_{t} v_{2, h}^{n}, \chi_{v, h}^{2}\right)+\gamma \Lambda\left(\bar{v}_{1, h}^{n-\frac{1}{2}}, \chi_{v, h}^{2}\right)-\lambda\left(\hat{G}_{v}^{n-\frac{1}{2}} \bar{v}_{1, h}^{n-\frac{1}{2}}, \chi_{v, h}^{2}\right)=0,
\end{aligned}
$$

subjected to the initial values

$$
\begin{array}{ll}
u_{1, h}^{0}=U_{1}^{0}(x), & u_{2, h}^{0}=U_{2}^{0}(x), \\
v_{1, h}^{0}=V_{1}^{0}(x), & v_{2, h}^{0}=V_{2}^{0}(x),
\end{array}
$$

where

$$
\begin{aligned}
& \hat{G}_{u}^{n-\frac{1}{2}}=\left|\hat{u}_{1, h}^{n-\frac{1}{2}}\right|^{2}+\left|\hat{u}_{2, h}^{n-\frac{1}{2}}\right|^{2}+\rho\left|\hat{v}_{1, h}^{n-\frac{1}{2}}\right|^{2}+\rho\left|\hat{v}_{2, h}^{n-\frac{1}{2}}\right|^{2}, \\
& \hat{G}_{v}^{n-\frac{1}{2}}=\rho\left|\hat{u}_{1, h}^{n-\frac{1}{2}}\right|^{2}+\rho\left|\hat{u}_{2, h}^{n-\frac{1}{2}}\right|^{2}+\left|\hat{v}_{1, h}^{n-\frac{1}{2}}\right|^{2}+\left|\hat{v}_{2, h}^{n-\frac{1}{2}}\right|^{2} .
\end{aligned}
$$

As for the starting data $\hat{u}_{1, h}^{\frac{1}{2}}, \hat{u}_{2, h}^{\frac{1}{2}}, \hat{v}_{1, h}^{\frac{1}{2}}, \hat{v}_{2, h}^{\frac{1}{2}}$, we compute them by the schemes

$$
\begin{aligned}
& \left(\frac{\hat{u}_{1, h}^{\frac{1}{2}}-u_{1, h}^{0}}{\tau / 2}, \chi_{u, h}^{1}\right)-\gamma \Lambda\left(\hat{u}_{2, h}^{\frac{1}{2}}, \chi_{u, h}^{1}\right)+\lambda\left(G_{u}^{0} \hat{u}_{2, h}^{\frac{1}{2}}, \chi_{u, h}^{1}\right)=0, \\
& \left(\frac{\hat{u}_{2, h}^{\frac{1}{2}}-u_{2, h}^{0}}{\tau / 2}, \chi_{u, h}^{2}\right)+\gamma \Lambda\left(\hat{u}_{1, h}^{\frac{1}{2}}, \chi_{u, h}^{2}\right)-\lambda\left(G_{u}^{0} \hat{u}_{1, h}^{\frac{1}{2}}, \chi_{u, h}^{2}\right)=0, \\
& \left(\frac{\hat{v}_{1, h}^{\frac{1}{2}}-v_{1, h}^{0}}{\tau / 2}, \chi_{v, h}^{1}\right)-\gamma \Lambda\left(\hat{v}_{2, h}^{\frac{1}{2}}, \chi_{v, h}^{1}\right)+\lambda\left(G_{v}^{0} \hat{v}_{2, h}^{\frac{1}{2}}, \chi_{v, h}^{1}\right)=0 . \\
& \left(\frac{\hat{v}_{2, h}^{\frac{1}{2}}-v_{2, h}^{0}}{\tau / 2}, \chi_{v, h}^{2}\right)+\gamma \Lambda\left(\hat{v}_{1, h}^{\frac{1}{2}}, \chi_{v, h}^{2}\right)-\lambda\left(G_{v}^{0} \hat{v}_{1, h}^{\frac{1}{2}}, \chi_{v, h}^{2}\right)=0,
\end{aligned}
$$

where

$$
\begin{aligned}
& G_{u}^{0}=\left|u_{1, h}^{0}\right|^{2}+\left|u_{2, h}^{0}\right|^{2}+\rho\left|v_{1, h}^{0}\right|^{2}+\rho\left|v_{2, h}^{0}\right|^{2}, \\
& G_{v}^{0}=\rho\left|u_{1, h}^{0}\right|^{2}+\rho\left|u_{2, h}^{0}\right|^{2}+\left|v_{1, h}^{0}\right|^{2}+\left|v_{2, h}^{0}\right|^{2} .
\end{aligned}
$$

It is evident that Eqs. (4.1)-(4.10) are decoupled by marching in time alternately with $u_{h}^{n}, v_{h}^{n}$ during the whole steps, which implies that at each temporal level, an array of linear system is only needed to be handled. This merit would be greatly beneficial to cutting the computing costs. In addition, the obtained discrete system can well preserve the mass in the discrete sense, given as follows.

Theorem 4.1 Let $u_{1, h}^{n}, u_{2, h}^{n}, v_{1, h}^{n}, v_{2, h}^{n}$ be the solutions obtained by the fully discrete FEM (4.1)-(4.10). Then, for $n=1,2, \ldots, N$, one has the mass conservation

$$
Q_{u}^{n}=Q_{u}^{0}, \quad Q_{v}^{n}=Q_{v}^{0},
$$

where $Q_{u}^{n}=\sqrt{\left\|u_{1, h}^{n}\right\|_{0, \Omega}^{2}+\left\|u_{2, h}^{n}\right\|_{0, \Omega}^{2}}$ and $Q_{v}^{n}=\sqrt{\left\|v_{1, h}^{n}\right\|_{0, \Omega}^{2}+\left\|v_{2, h}^{n}\right\|_{0, \Omega}^{2}}$. 
Proof Taking $\chi_{u, h}^{1}=\bar{u}_{1, h}^{n-\frac{1}{2}}$ in Eq. (4.1) and $\chi_{u, h}^{2}=\bar{u}_{2, h}^{n-\frac{1}{2}}$ in Eq. (4.2), we obtain

$$
\begin{aligned}
& \left(\bar{\partial}_{t} u_{1, h}^{n}, \bar{u}_{1, h}^{n-\frac{1}{2}}\right)-\gamma \Lambda\left(\bar{u}_{2, h}^{n-\frac{1}{2}}, \bar{u}_{1, h}^{n-\frac{1}{2}}\right)+\lambda\left(\hat{G}_{u}^{n-\frac{1}{2}} \bar{u}_{2, h}^{n-\frac{1}{2}}, \bar{u}_{1, h}^{n-\frac{1}{2}}\right)=0, \\
& \left(\bar{\partial}_{t} u_{2, h}^{n}, \bar{u}_{2, h}^{n-\frac{1}{2}}\right)+\gamma \Lambda\left(\bar{u}_{1, h}^{n-\frac{1}{2}}, \bar{u}_{2, h}^{n-\frac{1}{2}}\right)-\lambda\left(\hat{G}_{u}^{n-\frac{1}{2}} \bar{u}_{1, h}^{n-\frac{1}{2}}, \bar{u}_{2, h}^{n-\frac{1}{2}}\right)=0 .
\end{aligned}
$$

Summing Eqs. (4.11)-(4.12), it follows from Theorem 3.1 that

$$
\left(\bar{\partial}_{t} u_{1, h}^{n}, \bar{u}_{1, h}^{n-\frac{1}{2}}\right)+\left(\bar{\partial}_{t} u_{2, h}^{n}, \bar{u}_{2, h}^{n-\frac{1}{2}}\right)=0
$$

which implies

$$
\left\|u_{1, h}^{n}\right\|_{0, \Omega}^{2}+\left\|u_{2, h}^{n}\right\|_{0, \Omega}^{2}=\left\|u_{1, h}^{n-1}\right\|_{0, \Omega}^{2}+\left\|u_{2, h}^{n-1}\right\|_{0, \Omega}^{2}
$$

and thus $Q_{u}^{n}=Q_{u}^{n-1}=\cdots=Q_{u}^{0}$. Letting $\chi_{v, h}^{1}=\bar{v}_{1, h}^{n-\frac{1}{2}}$ in Eq. (4.3) and $\chi_{v, h}^{2}=\bar{v}_{2, h}^{n-\frac{1}{2}}$ in Eq. (4.4), repeating the processes above leads to the argument $Q_{v}^{n}=Q_{v}^{0}$. This concludes the conservative properties.

\section{Convergent analysis}

In this section, we derive the convergent estimate for the linearized Crank-Nicolson FEM (4.1)-(4.10). We shall start by introducing a fractional orthogonal projection: $\mathcal{R}_{h}$ : $H_{0}^{\alpha}(\Omega) \rightarrow \mathcal{V}_{h}$, which is defined by

$$
\Lambda\left(u, v_{h}\right)=\Lambda\left(\mathcal{R}_{h} u, v_{h}\right), \quad \forall v_{h} \in \mathcal{V}_{h}
$$

In view of $[17,55]$, the following properties are admitted.

Lemma 5.1 If $u \in H_{0}^{\alpha}(\Omega) \cap H^{k}(\Omega)$, then we have, for $\mathcal{R}_{h}$, that

$$
\begin{aligned}
& \left\|u-\mathcal{R}_{h} u\right\|_{0, \Omega} \lesssim h^{k}\|u\|_{k, \Omega}, \quad \alpha \neq 3 / 4 \\
& \left\|u-\mathcal{R}_{h} u\right\|_{0, \Omega} \lesssim h^{k-\epsilon}\|u\|_{k, \Omega}, \quad \alpha=3 / 4,0<\epsilon<1 / 2 .
\end{aligned}
$$

In what follows, we turn to the convergence of FEM (4.1)-(4.10) based on operator $\mathcal{R}_{h}$. Let $u^{n}, v^{n}$ be the analytic solutions to Eqs. (1.1)-(1.4) at $t=t_{n}$ with their real and imaginary parts $u_{1}^{n}, u_{2}^{n}, v_{1}^{n}, v_{2}^{n}$ respectively, i.e., $u^{n}=u_{1}^{n}+\mathrm{i} u_{2}^{n}, v^{n}=v_{1}^{n}+\mathrm{i} v_{2}^{n}$, and $u_{1, h}^{n}$, $u_{2, h}^{n}, v_{1, h}^{n}, v_{2, h}^{n}$ are the numerical solutions obtained by FEM (4.1)-(4.10). Also, we define $u_{h}^{n}=u_{1, h}^{n}+\mathrm{i} u_{2, h}^{n}, v_{h}^{n}=v_{1, h}^{n}+\mathrm{i} v_{2, h}^{n}$, and the complex norms $\left\|u_{h}^{n}\right\|_{0, \Omega}=\sqrt{\left\|u_{1, h}^{n}\right\|_{0, \Omega}^{2}+\left\|u_{2, h}^{n}\right\|_{0, \Omega}^{2}}$, $\left\|v_{h}^{n}\right\|_{0, \Omega}=\sqrt{\left\|v_{1, h}^{n}\right\|_{0, \Omega}^{2}+\left\|v_{2, h}^{n}\right\|_{0, \Omega}^{2}}$. Owing to Taylor expansions, we have

$$
\begin{aligned}
&\left\|\partial_{t} f^{n-\frac{1}{2}}-\bar{\partial}_{t} f^{n}\right\|_{0, \Omega} \lesssim \tau^{2}\left\|\partial_{t}^{3} f\right\|_{L^{\infty}\left(t_{n-1}, t_{n}, L^{2}\right)} \\
&\left\|f^{n-\frac{1}{2}}-\bar{f}^{n-\frac{1}{2}}\right\|_{p, \Omega} \lesssim \tau^{2}\left\|\partial_{t}^{2} f\right\|_{L^{\infty}\left(t_{n-1}, t_{n}, H^{p}\right)}, \\
&\left\|f^{n-\frac{1}{2}}-\hat{f}^{n-\frac{1}{2}}\right\|_{0, \Omega} \lesssim \tau^{2}\left\|\partial_{t}^{2} f\right\|_{L^{\infty}\left(t_{n-2}, t_{n}, L^{2}\right)}, \quad n \geq 2,
\end{aligned}
$$


if $\partial_{t}^{3} f \in L^{\infty}\left(t_{n-1}, t_{n}, L^{2}\right), \partial_{t}^{2} f \in L^{\infty}\left(t_{n-1}, t_{n}, H^{p}\right)$, and $\partial_{t}^{2} f \in L^{\infty}\left(t_{n-2}, t_{n}, L^{2}\right)$. Let $e_{u}^{n}=u^{n}-u_{h}^{n}=$ $\rho_{u}^{n}-\theta_{u}^{n}, \rho_{u}^{n}=u^{n}-\mathcal{R}_{h} u^{n}, \theta_{u}^{n}=u_{h}^{n}-\mathcal{R}_{h} u^{n}$, and $u_{j}^{n}-u_{j, h}^{n}=\rho_{u_{j}}^{n}-\theta_{u_{j}}^{n}, \bar{u}_{j}^{n-\frac{1}{2}}-\bar{u}_{j, h}^{n-\frac{1}{2}}=\bar{\rho}_{u_{j}}^{n-\frac{1}{2}}-\bar{\theta}_{u_{j}}^{n-\frac{1}{2}}$, with $j=1,2$, and

$$
\begin{aligned}
& \rho_{u_{j}}^{n}=u_{j}^{n}-\mathcal{R}_{h} u_{j}^{n}, \quad \theta_{u_{j}}^{n}=u_{j, h}^{n}-\mathcal{R}_{h} u_{j, h}^{n}, \\
& \bar{\rho}_{u_{j}}^{n-\frac{1}{2}}=\bar{u}_{j}^{n-\frac{1}{2}}-\mathcal{R}_{h} \bar{u}_{j}^{n-\frac{1}{2}}, \quad \bar{\theta}_{u_{j}}^{n-\frac{1}{2}}=\bar{u}_{j, h}^{n-\frac{1}{2}}-\mathcal{R}_{h} \bar{u}_{j}^{n-\frac{1}{2}} .
\end{aligned}
$$

Then, by the aid of mathematical induction, the convergent theorem is admitted.

Theorem 5.1 Assume that

$$
\begin{aligned}
& \partial_{t}^{2} u_{1}, \partial_{t}^{2} u_{2} \in L^{\infty}\left(H^{k} \cap H^{2 \alpha}\right), \\
& u_{1}, u_{2}, \partial_{t} u_{1}, \partial_{t} u_{2}, \partial_{t}^{3} u_{1}, \partial_{t}^{3} u_{2} \in L^{\infty}\left(H^{k}\right),
\end{aligned}
$$

and the same conditions hold for $v_{1}, v_{2}$. Let $U_{1}^{0}=\mathcal{R}_{h} u_{1}^{0}, U_{2}^{0}=\mathcal{R}_{h} u_{2}^{0}, V_{1}^{0}=\mathcal{R}_{h} v_{1}^{0}, V_{2}^{0}=\mathcal{R}_{h} v_{2}^{0}$, and $\tau=\kappa h^{\frac{1}{4}+\delta}$, where $\kappa, \delta$ are positive numbers. Then, when $\alpha \neq 3 / 4$, one has

$$
\left\|u^{n}-u_{h}^{n}\right\|_{0, \Omega} \lesssim O\left(\tau^{2}+h^{k}\right), \quad\left\|v^{n}-v_{h}^{n}\right\|_{0, \Omega} \lesssim O\left(\tau^{2}+h^{k}\right),
$$

and when $\alpha=3 / 4$, one has

$$
\left\|u^{n}-u_{h}^{n}\right\|_{0, \Omega} \lesssim O\left(\tau^{2}+h^{k-\epsilon}\right), \quad\left\|v^{n}-v_{h}^{n}\right\|_{0, \Omega} \lesssim O\left(\tau^{2}+h^{k-\epsilon}\right)
$$

where $0<\epsilon<1 / 2$ and $n=0,1, \ldots, N$.

Proof It is clear that (5.7)-(5.8) are valid as $n=0$. Since Eqs. (4.1)-(4.10) are decoupled, we can proceed with the estimates individually by variables. For this reason, we are only concerned with the errors of $u_{1, h}^{n}, u_{2, h}^{n}$, and the results corresponding to $v_{1, h}^{n}, v_{2, h}^{n}$ follow. In the initial step, by Eqs. (3.5)-(3.6), $u_{1}^{\frac{1}{2}}, u_{2}^{\frac{1}{2}}$ solve

$$
\begin{aligned}
& \left(\frac{u_{1}^{\frac{1}{2}}-u_{1}^{0}}{\tau / 2}, \chi_{u, h}^{1}\right)-\gamma \Lambda\left(u_{2}^{\frac{1}{2}}, \chi_{u, h}^{1}\right)+\lambda\left(G^{0} u_{2}^{\frac{1}{2}}, \chi_{u, h}^{1}\right)+\left(\widehat{P}_{1}, \chi_{u, h}^{1}\right)=0, \\
& \left(\frac{u_{2}^{\frac{1}{2}}-u_{2}^{0}}{\tau / 2}, \chi_{u, h}^{2}\right)+\gamma \Lambda\left(u_{1}^{\frac{1}{2}}, \chi_{u, h}^{2}\right)-\lambda\left(G^{0} u_{1}^{\frac{1}{2}}, \chi_{u, h}^{2}\right)+\left(\widehat{P}_{2}, \chi_{u, h}^{2}\right)=0,
\end{aligned}
$$

where $G^{0}=\left|u_{1}^{0}\right|^{2}+\left|u_{2}^{0}\right|^{2}+\rho\left|v_{1}^{0}\right|^{2}+\rho\left|v_{2}^{0}\right|^{2}, \widehat{P}_{1}, \widehat{P}_{2}$ are the truncated errors in time, which satisfy $\left\|\widehat{P}_{1}\right\|_{0, \Omega},\left\|\widehat{P}_{2}\right\|_{0, \Omega} \lesssim O(\tau)$ by Taylor formula. Let $\widehat{\rho}_{u_{1}}^{\frac{1}{2}}=u_{1}^{\frac{1}{2}}-\mathcal{R}_{h} u_{1}^{\frac{1}{2}}, \widehat{\theta}_{u_{1}}^{\frac{1}{2}}=\hat{u}_{1, h}^{\frac{1}{2}}-\mathcal{R}_{h} u_{1}^{\frac{1}{2}}$, and $\hat{\rho}_{u_{2}}^{\frac{1}{2}}=u_{2}^{\frac{1}{2}}-\mathcal{R}_{h} u_{2}^{\frac{1}{2}}, \widehat{\theta}_{u_{2}}^{\frac{1}{2}}=\hat{u}_{2, h}^{\frac{1}{2}}-\mathcal{R}_{h} u_{2}^{\frac{1}{2}}$. Then, subtracting Eqs. (4.7), (4.8) from Eqs. (5.9), (5.10), respectively, and using (5.1) lead to

$$
\begin{aligned}
& \left(\frac{\widehat{\theta}_{u_{1}}^{\frac{1}{2}}-\theta_{u_{1}}^{0}}{\tau / 2}, \chi_{u, h}^{1}\right)-\gamma \Lambda\left(\widehat{\theta}_{u_{2}}^{\frac{1}{2}}, \chi_{u, h}^{1}\right)=\left(\frac{\widehat{\rho}_{u_{1}}^{\frac{1}{2}}-\rho_{u_{1}}^{0}}{\tau / 2}, \chi_{u, h}^{1}\right)+\left(\widehat{G}_{2}, \chi_{u, h}^{1}\right)+\left(\widehat{P}_{1}, \chi_{u, h}^{1}\right), \\
& \left(\frac{\widehat{\theta}_{u_{2}}^{\frac{1}{2}}-\theta_{u_{2}}^{0}}{\tau / 2}, \chi_{u, h}^{2}\right)+\gamma \Lambda\left(\widehat{\theta}_{u_{1}}^{\frac{1}{2}}, \chi_{u, h}^{2}\right)=\left(\frac{\widehat{\rho}_{u_{2}}^{\frac{1}{2}}-\rho_{u_{2}}^{0}}{\tau / 2}, \chi_{u, h}^{2}\right)-\left(\widehat{G}_{1}, \chi_{u, h}^{2}\right)+\left(\widehat{P}_{2}, \chi_{u, h}^{2}\right),
\end{aligned}
$$


in which

$$
\begin{aligned}
\widehat{G}_{j} & =\lambda G^{0} u_{j}^{\frac{1}{2}}-\lambda G_{u}^{0} \hat{u}_{j, h}^{\frac{1}{2}} \\
& =\lambda\left(\left|u_{1}^{0}\right|^{2}+\left|u_{2}^{0}\right|^{2}+\rho\left|v_{1}^{0}\right|^{2}+\rho\left|v_{2}^{0}\right|^{2}\right) u_{j}^{\frac{1}{2}}-\lambda\left(\left|u_{1, h}^{0}\right|^{2}+\left|u_{2, h}^{0}\right|^{2}+\rho\left|v_{1, h}^{0}\right|^{2}+\rho\left|v_{2, h}^{0}\right|^{2}\right) \hat{u}_{j, h}^{\frac{1}{2}} .
\end{aligned}
$$

Setting $\chi_{u, h}^{1}=\widehat{\theta}_{u_{1}}^{\frac{1}{2}}$ in Eq. (5.11), $\chi_{u, h}^{2}=\widehat{\theta}_{u_{2}}^{\frac{1}{2}}$ in Eq. (5.12) and adding together, one has

$$
\begin{aligned}
\frac{\left\|\widehat{\theta}_{u_{1}}^{\frac{1}{2}}\right\|_{0, \Omega}^{2}+\left\|\widehat{\theta}_{u_{2}}^{\frac{1}{2}}\right\|_{0, \Omega}^{2}}{\tau / 2} & \left(\frac{\widehat{\rho}_{u_{1}}^{\frac{1}{2}}-\rho_{u_{1}}^{0}}{\tau / 2}, \widehat{\theta}_{u_{1}}^{\frac{1}{2}}\right)+\left(\frac{\widehat{\rho}_{u_{2}}^{\frac{1}{2}}-\rho_{u_{2}}^{0}}{\tau / 2}, \widehat{\theta}_{u_{2}}^{\frac{1}{2}}\right) \\
& +\left(\widehat{G}_{2}, \widehat{\theta}_{u_{1}}^{\frac{1}{2}}\right)-\left(\widehat{G}_{1}, \widehat{\theta}_{u_{2}}^{\frac{1}{2}}\right)+\left(\widehat{P}_{1}, \widehat{\theta}_{u_{1}}^{\frac{1}{2}}\right)+\left(\widehat{P}_{2}, \widehat{\theta}_{u_{2}}^{\frac{1}{2}}\right) \\
\leq & \left\|\frac{\widehat{\rho}_{u_{1}}^{\frac{1}{2}}-\rho_{u 1}^{0}}{\tau / 2}\right\|_{0, \Omega}\left\|\widehat{\theta}_{u_{1}}^{\frac{1}{2}}\right\|_{0, \Omega}+\left|\frac{\widehat{\rho}_{u_{2}}^{\frac{1}{2}}-\rho_{u 2}^{0}}{\tau / 2}\right|_{0, \Omega}\left\|\widehat{\theta}_{u_{2}}^{\frac{1}{2}}\right\|_{0, \Omega} \\
& +\left\|\widehat{G}_{1}\right\|_{0, \Omega}\left\|\widehat{\theta}_{u_{2}}^{\frac{1}{2}}\right\|_{0, \Omega}+\left\|\widehat{G}_{2}\right\|_{0, \Omega}\left\|\widehat{\theta}_{u_{1}}^{\frac{1}{2}}\right\|_{0, \Omega} \\
& +\left\|\widehat{P}_{1}\right\|_{0, \Omega}\left\|\widehat{\theta}_{u_{1}}^{\frac{1}{2}}\right\|_{0, \Omega}+\left\|\widehat{P}_{2}\right\|_{0, \Omega}\left\|\widehat{\theta}_{u_{2}}^{\frac{1}{2}}\right\|_{0, \Omega} \\
\leq & C \tau\left\{\left\|\frac{\hat{\rho}_{u_{1}}^{\frac{1}{2}}-\rho_{u_{1}}^{0} \|^{2}}{\tau / 2}\right\|_{0, \Omega}^{2}\left\|\frac{\hat{\rho}_{u_{2}}^{\frac{1}{2}}-\rho_{u_{2}}^{0} \|^{2}}{\tau / 2}\right\|_{0, \Omega}^{2}+\left\|\widehat{G}_{1}\right\|_{0, \Omega}^{2}+\left\|\widehat{G}_{2}\right\|_{0, \Omega}^{2}\right. \\
& \left.+\left\|\widehat{P}_{1}\right\|_{0, \Omega}^{2}+\left\|\widehat{P}_{2}\right\|_{0, \Omega}^{2}\right\}+\frac{1}{4 \tau}\left\{\left\|\widehat{\theta}_{u_{1}}^{\frac{1}{2}}\right\|_{0, \Omega}^{2}+\left\|\widehat{\theta}_{u_{2}}^{\frac{1}{2}}\right\|_{0, \Omega}^{2}\right\},
\end{aligned}
$$

where $\gamma \Lambda\left(\widehat{\theta}_{u_{1}}^{\frac{1}{2}}, \widehat{\theta}_{u_{2}}^{\frac{1}{2}}\right)$ vanishes due to Theorem 3.1. It follows from Lemma 5.1 that

$$
\begin{gathered}
\left\|\frac{\hat{\rho}_{u_{1}}^{\frac{1}{2}}-\rho_{u_{1}}^{0}}{\tau / 2}\right\|_{0, \Omega}^{2} \lesssim\left\|\partial_{t} u_{1}-\partial_{t} \mathcal{R}_{h} u_{1}\right\|_{L^{\infty}\left(0, t_{1 / 2} ; L^{2}\right)}^{2} \lesssim O\left(h^{2 \tilde{k}}\right), \\
\left\|\frac{\widehat{\rho}_{u_{2}}^{\frac{1}{2}}-\rho_{u_{2}}^{0}}{\tau / 2}\right\|_{0, \Omega}^{2} \lesssim\left\|\partial_{t} u_{2}-\partial_{t} \mathcal{R}_{h} u_{2}\right\|_{L^{\infty}\left(0, t_{1 / 2} ; L^{2}\right)}^{2} \lesssim O\left(h^{2 \tilde{k}}\right), \\
\left\|\widehat{G}_{1}\right\|_{0, \Omega}^{2} \lesssim\left\|\left|u_{1}^{0}\right|^{2} u_{1}^{\frac{1}{2}}-\left|u_{1, h}^{0}\right|^{2} u_{1, h}^{\frac{1}{2}}+\left|u_{2}^{0}\right|^{2} u_{1}^{\frac{1}{2}}-\left|u_{2, h}^{0}\right|^{2} \hat{u}_{1, h}^{\frac{1}{2}}\right\|_{0, \Omega}^{2} \\
+\rho^{2}\left\|\left|v_{1}^{0}\right|^{2} u_{1}^{\frac{1}{2}}-\left|v_{1, h}^{0}\right|^{2} \hat{u}_{1, h}^{\frac{1}{2}}+\left|v_{2}^{0}\right|^{2} u_{1}^{\frac{1}{2}}-\left|v_{2, h}^{0}\right|^{2} \hat{u}_{1, h}^{\frac{1}{2}}\right\|_{0, \Omega}^{2} \\
\lesssim O\left(h^{2 \tilde{k}}\right)+\left\|\widehat{\theta}_{u_{1}}^{\frac{1}{2}}\right\|_{0, \Omega}^{2}, \\
\left\|\widehat{G}_{2}\right\|_{0, \Omega}^{2} \lesssim\left\|\left|u_{1}^{0}\right|^{2} u_{2}^{\frac{1}{2}}-\left|u_{1, h}^{0}\right|^{2} u_{2, h}^{\frac{1}{2}}+\left|u_{2}^{0}\right|^{2} u_{2}^{\frac{1}{2}}-\left|u_{2, h}^{0}\right|^{2} \hat{u}_{2, h}^{\frac{1}{2}}\right\|_{0, \Omega}^{2} \\
+\rho^{2}\left\|\left|v_{1}^{0}\right|^{2} u_{2}^{\frac{1}{2}}-\left|v_{1, h}^{0}\right|^{2} \hat{u}_{2, h}^{\frac{1}{2}}+\left|v_{2}^{0}\right|^{2} u_{2}^{\frac{1}{2}}-\left|v_{2, h}^{0}\right|^{2} \hat{u}_{2, h}^{\frac{1}{2}}\right\|_{0, \Omega}^{2} \\
\lesssim O\left(h^{2 \tilde{k}}\right)+|| \widehat{\theta}_{u_{2}}^{\frac{1}{2}} \|_{0, \Omega}^{2},
\end{gathered}
$$


where if $\alpha \neq 3 / 4, \tilde{k}=k$ and if $\alpha=3 / 4, \tilde{k}=k-\epsilon, 0<\epsilon<1 / 2$. Inserting (5.14)-(5.17) into (5.13), we get

$$
\left\|\widehat{\theta}_{u}^{\frac{1}{2}}\right\|_{0, \Omega}^{2}=\left\|\widehat{\theta}_{u_{1}}^{\frac{1}{2}}\right\|_{0, \Omega}^{2}+\left\|\widehat{\theta}_{u_{2}}^{\frac{1}{2}}\right\|_{0, \Omega}^{2} \lesssim O\left(\tau^{4}+h^{2 \tilde{k}}\right)
$$

by opting for a small enough $\tau$, and thus

$$
\left\|\hat{e}_{u}^{1}\right\|_{0, \Omega}^{2}=\left\|u^{\frac{1}{2}}-\hat{u}_{h}^{\frac{1}{2}}\right\|_{0, \Omega}^{2} \lesssim O\left(\tau^{4}+h^{2 \tilde{k}}\right) .
$$

As to $n=1$, using the inverse inequality, one gets

$$
\left\|\widehat{\boldsymbol{e}}_{u}^{1}\right\|_{\infty, \Omega} \lesssim h^{-\frac{1}{2}}\left\|\widehat{\boldsymbol{e}}_{u}^{1}\right\|_{0, \Omega} \lesssim O\left(\tau^{2} h^{-\frac{1}{2}}+h^{\tilde{k}-\frac{1}{2}}\right) \lesssim O\left(\kappa^{2} h^{2 \delta}+h^{\tilde{k}-\frac{1}{2}}\right)
$$

in the sense that $\left\|\hat{u}_{1, h}^{\frac{1}{2}}\right\|_{\infty, \Omega},\left\|\hat{u}_{2, h}^{\frac{1}{2}}\right\|_{\infty, \Omega}$ are bounded. The same results are valid for $\left\|\hat{v}_{1, h}^{\frac{1}{2}}\right\|_{\infty, \Omega},\left\|\hat{v}_{2, h}^{\frac{1}{2}}\right\|_{\infty, \Omega}$. Analogously, via Eq. (5.1), the difference of Eqs. (4.1)-(4.2) and Eqs. (3.5)-(3.6) gives

$$
\begin{aligned}
& \left(\bar{\partial}_{t} \theta_{u_{1}}^{\frac{1}{2}}, \bar{\theta}_{u_{1}}^{\frac{1}{2}}\right)-\gamma \Lambda\left(\bar{\theta}_{u_{2}}^{\frac{1}{2}}, \bar{\theta}_{u_{1}}^{\frac{1}{2}}\right)=\left(\partial_{t} u_{1}^{\frac{1}{2}}-\bar{\partial}_{t} \mathcal{R}_{h} u_{1}^{\frac{1}{2}}, \bar{\theta}_{u_{1}}^{\frac{1}{2}}\right)+\gamma\left(\partial_{x}^{2 \alpha} F_{2}^{1}, \bar{\theta}_{u_{1}}^{\frac{1}{2}}\right)+\left(G_{2}^{1}, \bar{\theta}_{u_{1}}^{\frac{1}{2}}\right), \\
& \left(\bar{\partial}_{t} \theta_{u_{2}}^{\frac{1}{2}}, \bar{\theta}_{u_{2}}^{\frac{1}{2}}\right)+\gamma \Lambda\left(\bar{\theta}_{u_{1}}^{\frac{1}{2}}, \bar{\theta}_{u_{2}}^{\frac{1}{2}}\right)=\left(\partial_{t} u_{2}^{\frac{1}{2}}-\bar{\partial}_{t} \mathcal{R}_{h} u_{2}^{\frac{1}{2}}, \bar{\theta}_{u_{2}}^{\frac{1}{2}}\right)-\gamma\left(\partial_{x}^{2 \alpha} F_{1}^{1}, \bar{\theta}_{u_{2}}^{\frac{1}{2}}\right)-\left(G_{1}^{1}, \bar{\theta}_{u_{2}}^{\frac{1}{2}}\right),
\end{aligned}
$$

in which

$$
\begin{aligned}
F_{j}^{1}= & u_{j}^{\frac{1}{2}}-\bar{u}_{j}^{\frac{1}{2}}, \\
G_{j}^{1}= & \lambda\left(\left|u_{1}^{\frac{1}{2}}\right|^{2}+\left|u_{2}^{\frac{1}{2}}\right|^{2}+\rho\left|v_{1}^{\frac{1}{2}}\right|^{2}+\rho\left|v_{2}^{\frac{1}{2}}\right|^{2}\right) u_{j}^{\frac{1}{2}} \\
& -\lambda\left(\left|\hat{u}_{1, h}^{\frac{1}{2}}\right|^{2}+\left|\hat{u}_{2, h}^{\frac{1}{2}}\right|^{2}+\rho\left|\hat{v}_{1, h}^{\frac{1}{2}}\right|^{2}+\rho\left|\hat{v}_{2, h}^{\frac{1}{2}}\right|^{2}\right) \bar{u}_{j, h}^{\frac{1}{2}},
\end{aligned}
$$

with $j=1,2$. In view of $\theta_{u_{j}}^{0}=0$, adding these two equations together yields

$$
\begin{aligned}
\frac{\left\|\theta_{u_{1}}^{1}\right\|_{0, \Omega}^{2}+\left\|\theta_{u_{2}}^{1}\right\|_{0, \Omega}^{2}}{\tau} & =\left(\partial_{t} u_{1}^{\frac{1}{2}}-\bar{\partial}_{t} \mathcal{R}_{h} u_{1}^{\frac{1}{2}}, \theta_{u_{1}}^{1}\right)+\left(\partial_{t} u_{2}^{\frac{1}{2}}-\bar{\partial}_{t} \mathcal{R}_{h} u_{2}^{\frac{1}{2}}, \theta_{u_{2}}^{1}\right) \\
& -\gamma\left(\partial_{x}^{2 \alpha} F_{1}^{1}, \theta_{u_{2}}^{1}\right)+\gamma\left(\partial_{x}^{2 \alpha} F_{2}^{1}, \theta_{u_{1}}^{1}\right)-\left(G_{1}^{1}, \theta_{u_{2}}^{1}\right)+\left(G_{2}^{1}, \theta_{u_{1}}^{1}\right) \\
\lesssim & \left\|\partial_{t} u_{1}^{\frac{1}{2}}-\bar{\partial}_{t} \mathcal{R}_{h} u_{1}^{\frac{1}{2}}\right\|_{0, \Omega}\left\|\theta_{u_{1}}^{1}\right\|_{0, \Omega}+\left\|\partial_{t} u_{2}^{\frac{1}{2}}-\bar{\partial}_{t} \mathcal{R}_{h} u_{2}^{\frac{1}{2}}\right\|_{0, \Omega}\left\|\theta_{u_{2}}^{1}\right\|_{0, \Omega} \\
& +\left\|\partial_{x}^{2 \alpha} F_{1}^{1}\right\|_{0, \Omega}\left\|\theta_{u_{2}}^{1}\right\|_{0, \Omega}+\left\|\partial_{x}^{2 \alpha} F_{2}^{1}\right\|_{0, \Omega}\left\|\theta_{u_{1}}^{1}\right\|_{0, \Omega} \\
& +\left\|G_{1}^{1}\right\|_{0, \Omega}\left\|\theta_{u_{2}}^{1}\right\|_{0, \Omega}+\left\|G_{2}^{1}\right\|_{0, \Omega}\left\|\theta_{u_{1}}^{1}\right\|_{0, \Omega} \\
\leq & C\left\{\left\|\partial_{t} u_{1}^{\frac{1}{2}}-\bar{\partial}_{t} \mathcal{R}_{h} u_{1}^{\frac{1}{2}}\right\|_{0, \Omega}^{2}+\left\|\partial_{t} u_{2}^{\frac{1}{2}}-\bar{\partial}_{t} \mathcal{R}_{h} u_{2}^{\frac{1}{2}}\right\|_{0, \Omega}^{2}\right. \\
& \left.+\left\|\partial_{x}^{2 \alpha} F_{1}^{1}\right\|_{0, \Omega}^{2}+\left\|\partial_{x}^{2 \alpha} F_{2}^{1}\right\|_{0, \Omega}^{2}+\left\|G_{1}^{1}\right\|_{0, \Omega}^{2}+\left\|G_{2}^{1}\right\|_{0, \Omega}^{2}\right\} \\
& +\frac{1}{2}\left\{\left\|\theta_{u_{1}}^{1}\right\|_{0, \Omega}^{2}+\left\|\theta_{u_{2}}^{1}\right\|_{0, \Omega}^{2}\right\} .
\end{aligned}
$$


Then, by Lemmas 2.3, 2.4, 5.1 and (5.4)-(5.6), we get

$$
\begin{aligned}
&\left\|\partial_{t} u_{j}^{\frac{1}{2}}-\bar{\partial}_{t} \mathcal{R}_{h} u_{j}^{\frac{1}{2}}\right\|_{0, \Omega}^{2} \lesssim\left\|\partial_{t} u_{j}^{\frac{1}{2}}-\bar{\partial}_{t} u_{j}^{\frac{1}{2}}\right\|_{0, \Omega}^{2}+\left\|\frac{\rho_{u_{j}}^{1}-\rho_{u_{j}}^{0}}{\tau}\right\|_{0, \Omega}^{2} \\
& \lesssim \tau^{4}\left\|\partial_{t}^{3} u_{j}\right\|_{L^{\infty}\left(0, t_{1} ; L^{2}\right)}^{2}+\left\|\partial_{t} u_{j}-\partial_{t} \mathcal{R}_{h} u_{j}\right\|_{L^{\infty}\left(0, t_{1} ; L^{2}\right)}^{2} \\
& \lesssim\left(\tau^{4}+h^{2 \tilde{k}}\right), \\
&\left\|\partial_{x}^{2 \alpha} F_{j}^{1}\right\|_{0, \Omega}^{2} \lesssim\left\|D_{L}^{2 \alpha} F_{j}^{1}\right\|_{0, \Omega}^{2}+\left\|D_{R}^{2 \alpha} F_{j}^{1}\right\|_{0, \Omega}^{2}=\left|F_{j}^{1}\right|_{L_{L}^{2 \alpha}(\Omega)}^{2}+\left|F_{j}^{1}\right|_{j_{R}^{2 \alpha}(\Omega)}^{2} \\
& \lesssim\left|F_{j}^{1}\right|_{2 \alpha, \Omega}^{2} \lesssim\left\|u_{j}^{\frac{1}{2}}-\bar{u}_{j}^{\frac{1}{2}}\right\|_{2 \alpha, \Omega}^{2} \lesssim O\left(\tau^{4}\right), \\
&\left\|G_{j}^{1}\right\|_{0, \Omega}^{2} \lesssim\left\|\left|u_{1}^{\frac{1}{2}}\right|^{2} u_{j}^{\frac{1}{2}}-\left|\hat{u}_{1, h}^{\frac{1}{2}}\right|^{2} \bar{u}_{j, h}^{\frac{1}{2}}+\left|u_{2}^{\frac{1}{2}}\right|^{2} u_{j}^{\frac{1}{2}}-\left|\hat{u}_{2, h}^{\frac{1}{2}}\right|^{2} \bar{u}_{j, h}^{\frac{1}{2}}\right\|_{0, \Omega}^{2} \\
&+\rho^{2}\left\|\left|v_{1}^{\frac{1}{2}}\right|^{2} u_{j}^{\frac{1}{2}}-\left|\hat{v}_{1, h}^{\frac{1}{2}}\right|^{2} \bar{u}_{j, h}^{\frac{1}{2}}+\left|v_{2}^{\frac{1}{2}}\right|^{2} u_{j}^{\frac{1}{2}}-\left|\hat{v}_{2, h}^{\frac{1}{2}}\right|^{2} \bar{u}_{j, h}^{\frac{1}{2}}\right\|_{0, \Omega}^{2} \\
& \lesssim\left\|\hat{e}_{u_{1}}^{1}\right\|_{0, \Omega}^{2}+\left\|\hat{e}_{u_{2}}^{1}\right\|_{0, \Omega}^{2}+\left\|\hat{e}_{v_{1}}^{1}\right\|_{0, \Omega}^{2}+\left\|\hat{e}_{v_{2}}^{1}\right\|_{0, \Omega}^{2} \\
&+\left\|e_{u_{j}}^{1}\right\|_{0, \Omega}^{2}+O\left(\tau^{4}+h^{2 \tilde{k}}\right) \\
& \lesssim\left\|\theta_{u_{j}}^{1}\right\|_{0, \Omega}^{2}+O\left(\tau^{4}+h^{2 \tilde{k}}\right),
\end{aligned}
$$

where $\left\|\hat{e}_{u_{j}}^{1}\right\|_{0, \Omega}=\left\|u_{j}^{\frac{1}{2}}-u_{j, h}^{\frac{1}{2}}\right\|_{0, \Omega},\left\|\widehat{e}_{v_{j}}^{1}\right\|_{0, \Omega}=\left\|v_{j}^{\frac{1}{2}}-v_{j, h}^{\frac{1}{2}}\right\|_{0, \Omega}$, and the boundedness of $\left\|\hat{u}_{j, h}^{\frac{1}{2}}\right\|_{\infty, \Omega}$, $\left\|\hat{v}_{j, h}^{\frac{1}{2}}\right\|_{\infty, \Omega}$ is applied. Inserting (5.19)-(5.21) into (5.18), we obtain

$$
\left\|\theta_{u}^{1}\right\|_{0, \Omega}^{2}=\left\|\theta_{u_{1}}^{1}\right\|_{0, \Omega}^{2}+\left\|\theta_{u_{2}}^{1}\right\|_{0, \Omega}^{2} \lesssim O\left(\tau^{4}+h^{2 \tilde{k}}\right),
$$

with a sufficiently small $\tau$. Hence, $\left\|e_{u}^{1}\right\|_{0, \Omega}^{2} \lesssim O\left(\tau^{4}+h^{2 \tilde{k}}\right)$ is concluded.

As to $n=m, m>1$, supposing that (5.7)-(5.8) are fulfilled when $n=m-1$, we begin to consider such case by mathematical induction. In the light of $\tau=\kappa h^{\frac{1}{4}+\delta}$, for any $u_{h}^{l}$, $1 \leq l \leq m-1$, it follows that

$$
\left\|e_{u}^{l}\right\|_{\infty, \Omega} \lesssim h^{-\frac{1}{2}}\left\|e_{u}^{l}\right\|_{0, \Omega} \lesssim O\left(\tau^{2} h^{-\frac{1}{2}}+h^{\tilde{k}-\frac{1}{2}}\right) \lesssim O\left(\kappa^{2} h^{2 \delta}+h^{\tilde{k}-\frac{1}{2}}\right)
$$

i.e., $\left\|u_{1, h}^{l}\right\|_{\infty, \Omega},\left\|u_{2, h}^{l}\right\|_{\infty, \Omega}$ are bounded, so are $\left\|v_{1, h}^{j}\right\|_{\infty, \Omega},\left\|v_{2, h}^{j}\right\|_{\infty, \Omega}$. Then, subtracting Eqs. (4.1)-(4.2) from Eqs. (3.5)-(3.6) at $t_{m-\frac{1}{2}}$ gives residual equations

$$
\begin{aligned}
& \left(\bar{\partial}_{t} \theta_{u_{1}}^{m-\frac{1}{2}}, \bar{\theta}_{u_{1}}^{m-\frac{1}{2}}\right)-\gamma \Lambda\left(\bar{\theta}_{u_{2}}^{m-\frac{1}{2}}, \bar{\theta}_{u_{1}}^{m-\frac{1}{2}}\right)=\left(P_{1}^{m}, \bar{\theta}_{u_{1}}^{m-\frac{1}{2}}\right)+\gamma\left(\partial_{x}^{2 \alpha} F_{2}^{m}, \bar{\theta}_{u_{1}}^{m-\frac{1}{2}}\right)+\left(G_{2}^{m}, \bar{\theta}_{u_{1}}^{m-\frac{1}{2}}\right), \\
& \left(\bar{\partial}_{t} \theta_{u_{2}}^{m-\frac{1}{2}}, \bar{\theta}_{u_{2}}^{m-\frac{1}{2}}\right)+\gamma \Lambda\left(\bar{\theta}_{u_{1}}^{m-\frac{1}{2}}, \bar{\theta}_{u_{2}}^{m-\frac{1}{2}}\right)=\left(P_{2}^{m}, \bar{\theta}_{u_{2}}^{m-\frac{1}{2}}\right)-\gamma\left(\partial_{x}^{2 \alpha} F_{1}^{m}, \bar{\theta}_{u_{2}}^{m-\frac{1}{2}}\right)-\left(G_{1}^{m}, \bar{\theta}_{u_{2}}^{m-\frac{1}{2}}\right),
\end{aligned}
$$

in which

$$
\begin{aligned}
P_{j}^{m}= & \partial_{t} u_{j}^{m-\frac{1}{2}}-\bar{\partial}_{t} \mathcal{R}_{h} u_{j}^{m-\frac{1}{2}}, F_{j}^{m}=u_{j}^{m-\frac{1}{2}}-\bar{u}_{j}^{m-\frac{1}{2}}, \\
G_{j}^{m}= & \lambda\left(\left|u_{1}^{m-\frac{1}{2}}\right|^{2}+\left|u_{2}^{m-\frac{1}{2}}\right|^{2}+\rho\left|v_{1}^{m-\frac{1}{2}}\right|^{2}+\rho\left|v_{2}^{m-\frac{1}{2}}\right|^{2}\right) u_{j}^{m-\frac{1}{2}} \\
& -\lambda\left(\left|\hat{u}_{1, h}^{m-\frac{1}{2}}\right|^{2}+\left|\hat{u}_{2, h}^{m-\frac{1}{2}}\right|^{2}+\rho\left|\hat{v}_{1, h}^{m-\frac{1}{2}}\right|^{2}+\rho\left|\hat{v}_{2, h}^{m-\frac{1}{2}}\right|^{2}\right) \bar{u}_{j, h}^{m-\frac{1}{2}},
\end{aligned}
$$


where $j=1,2$. By the sum of residual equations and Theorem 3.1, we obtain

$$
\begin{aligned}
& \frac{\left\|\theta_{u_{1}}^{m}\right\|_{0, \Omega}^{2}+\left\|\theta_{u_{2}}^{m}\right\|_{0, \Omega}^{2}-\left\|\theta_{u_{1}}^{m-1}\right\|_{0, \Omega}^{2}-\left\|\theta_{u_{2}}^{m-1}\right\|_{0, \Omega}^{2}}{\tau / 2} \\
& =\left(P_{1}^{m}, \bar{\theta}_{u_{1}}^{m-\frac{1}{2}}\right)+\left(P_{2}^{m}, \bar{\theta}_{u_{2}}^{m-\frac{1}{2}}\right) \\
& \quad-\gamma\left(\partial_{x}^{2 \alpha} F_{1}^{m}, \bar{\theta}_{u_{2}}^{m-\frac{1}{2}}\right)+\gamma\left(\partial_{x}^{2 \alpha} F_{2}^{m}, \bar{\theta}_{u_{1}}^{m-\frac{1}{2}}\right)-\left(G_{1}^{m}, \bar{\theta}_{u_{2}}^{m-\frac{1}{2}}\right)+\left(G_{2}^{m}, \bar{\theta}_{u_{1}}^{m-\frac{1}{2}}\right) \\
& \lesssim\left\|P_{1}^{m}\right\|_{0, \Omega}^{2}+\left\|P_{2}^{m}\right\|_{0, \Omega}^{2}+\left\|\partial_{x}^{2 \alpha} F_{1}^{m}\right\|_{0, \Omega}^{2}+\left\|\partial_{x}^{2 \alpha} F_{2}^{m}\right\|_{0, \Omega}^{2} \\
& \quad+\left\|G_{1}^{m}\right\|_{0, \Omega}^{2}+\left\|G_{2}^{m}\right\|_{0, \Omega}^{2}+\left\|\bar{\theta}_{u_{1}}^{m-\frac{1}{2}}\right\|_{0, \Omega}^{2}+\left\|\bar{\theta}_{u_{2}}^{m-\frac{1}{2}}\right\|_{0, \Omega}^{2} .
\end{aligned}
$$

Further, via the foregoing discussions, we easily know

$$
\begin{aligned}
& \left\|P_{j}^{m}\right\|_{0, \Omega}^{2} \lesssim O\left(\tau^{4}+h^{2 \tilde{k}}\right), \quad\left\|\partial_{x}^{2 \alpha} F_{j}^{m}\right\|_{0, \Omega}^{2} \lesssim\left|F_{j}^{m}\right|_{2 \alpha, \Omega}^{2} \lesssim O\left(\tau^{4}\right), \\
& \left\|\bar{\theta}_{u_{j}}^{m-\frac{1}{2}}\right\|_{0, \Omega}^{2} \lesssim\left\|\theta_{u_{j}}^{m}\right\|_{0, \Omega}^{2}+\left\|\theta_{u_{j}}^{m-1}\right\|_{0, \Omega}^{2} \lesssim\left\|\theta_{u_{j}}^{m}\right\|_{0, \Omega}^{2}+O\left(\tau^{4}+h^{2 \tilde{k}}\right) .
\end{aligned}
$$

Regarding the term $\left\|G_{j}^{m}\right\|_{0, \Omega}^{2}$, we estimate it as

$$
\begin{aligned}
\left\|G_{j}^{m}\right\|_{0, \Omega}^{2} \lesssim & \left\|\left|u_{1}^{m-\frac{1}{2}}\right|^{2} u_{j}^{m-\frac{1}{2}}-\left|\hat{u}_{1, h}^{m-\frac{1}{2}}\right|^{2} \bar{u}_{j, h}^{m-\frac{1}{2}}+\left|u_{2}^{m-\frac{1}{2}}\right|^{2} u_{j}^{m-\frac{1}{2}}-\left|\hat{u}_{2, h}^{m-\frac{1}{2}}\right|^{2} \bar{u}_{j, h}^{m-\frac{1}{2}}\right\|_{0, \Omega}^{2} \\
& +\rho^{2}\left\|\left|v_{1}^{m-\frac{1}{2}}\right|^{2} u_{j}^{m-\frac{1}{2}}-\left|\hat{v}_{1, h}^{m-\frac{1}{2}}\right|^{2} \bar{u}_{j, h}^{m-\frac{1}{2}}+\left|v_{2}^{m-\frac{1}{2}}\right|^{2} u_{j}^{m-\frac{1}{2}}-\left|\hat{v}_{2, h}^{m-\frac{1}{2}}\right|^{2} \bar{u}_{j, h}^{m-\frac{1}{2}}\right\|_{0, \Omega}^{2} \\
\lesssim & \left\|\theta_{u_{1}}^{m}\right\|_{0, \Omega}^{2}+\left\|\theta_{u_{2}}^{m}\right\|_{0, \Omega}^{2}+O\left(\tau^{4}+h^{2 \tilde{k}}\right),
\end{aligned}
$$

by (5.5)-(5.6) and the boundedness of $\left\|\hat{u}_{1, h}^{l}\right\|_{\infty, \Omega},\left\|\hat{u}_{2, h}^{l}\right\|_{\infty, \Omega},\left\|\hat{v}_{1, h}^{l}\right\|_{\infty, \Omega},\left\|\hat{v}_{2, h}^{l}\right\|_{\infty, \Omega}, 1 \leq l \leq$ $m-1$. Inserting (5.23)-(5.25) into (5.22) and applying Gronwall's inequality to the sum of the resulting (5.22) from 1 to $m$ with small $\tau$, we arrive at

$$
\left\|\theta_{u}^{m}\right\|_{0, \Omega}^{2}=\left\|\theta_{u_{1}}^{m}\right\|_{0, \Omega}^{2}+\left\|\theta_{u_{2}}^{m}\right\|_{0, \Omega}^{2} \lesssim O\left(\tau^{4}+h^{2 \tilde{k}}\right)
$$

where $\theta_{u_{1}}^{0}=0, \theta_{u_{2}}^{0}=0$ are used again. Hence, under Lemma 5.1, we get

$$
\left\|e_{u}^{m}\right\|_{0, \Omega}^{2} \lesssim\left\|\rho_{u}^{m}\right\|_{0, \Omega}^{2}+\left\|\theta_{u}^{m}\right\|_{0, \Omega}^{2} \lesssim O\left(\tau^{4}+h^{2 \tilde{k}}\right)
$$

In the same fashion, repeating the processes above can establish the desirable $\left\|e_{v}^{m}\right\|_{0, \Omega}^{2} \lesssim$ $O\left(\tau^{4}+h^{2 \tilde{k}}\right)$. The induction is completed.

Remarks 5.1 The restriction imposed on the temporal stepsize $\tau$ in Theorem 5.1 is just a need in theory. It is not necessary for us to comply with such a restriction in realistic implementation. Actually, it may be removed by the analytical technique derived in [56]; but it is beyond our scope to address this issue here.

\section{Numerical experiments}

In this part, numerical experiments are performed to support our theoretical results as well as gauge the actual performance of FEM (4.1)-(4.10). As stated earlier, the exact solutions to Eqs. (1.1)-(1.2) will polynomially or exponentially decay to zero when $|x|$ tends 

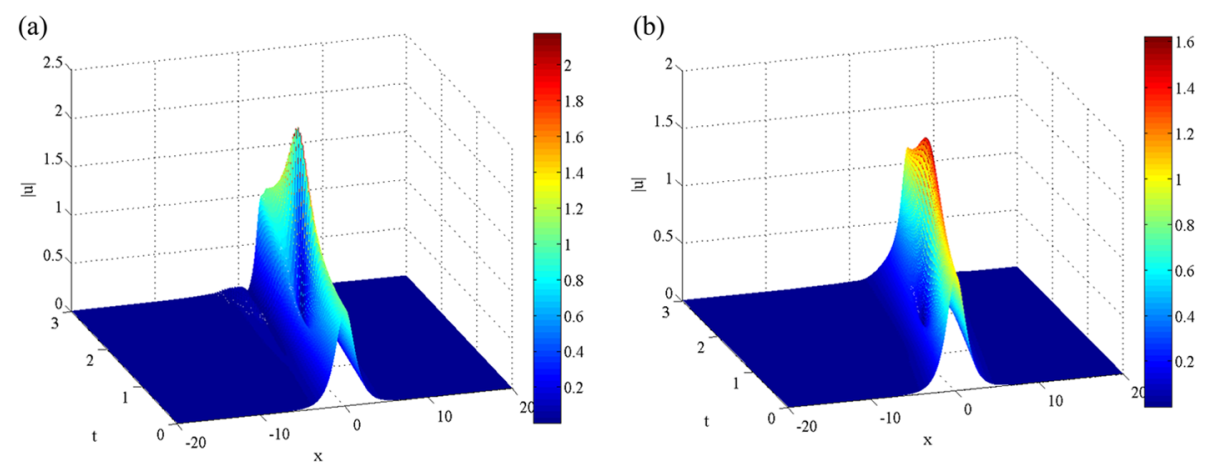

(c)

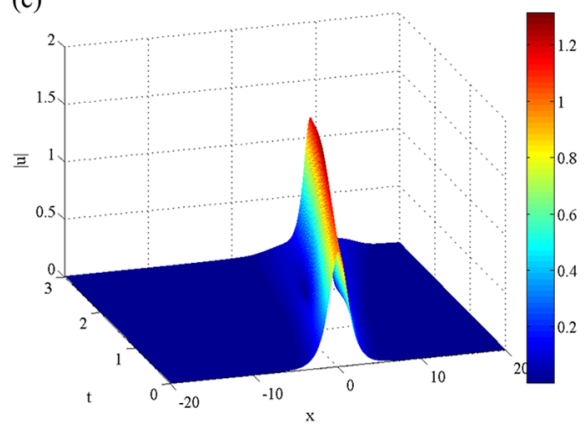

(d)

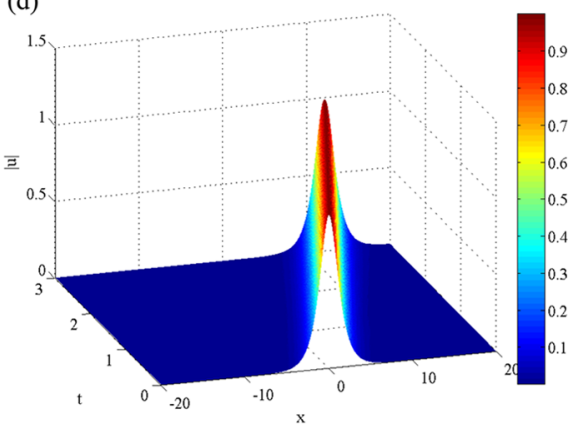

Figure 1 The wave propagations for different $\alpha$ with $\gamma=1, \lambda=2 ;(\mathbf{a}): \alpha=0.6,(\mathbf{b}): \alpha=0.8,(\mathbf{c}): \alpha=0.9$, (d): $\alpha=1$

to infinity, a large $\Omega$ will be truncated so that the wave functions are negligible outside of $\Omega$ and thereby (1.4) can successfully be enforced. In the tests below, we always set $\mathcal{V}_{h}$ to be a piecewise linear space.

Example 6.1 Let $\rho=0$. The model simply becomes

$$
\mathrm{i} \partial_{t} u+\gamma \partial_{x}^{2 \alpha} u+\lambda|u|^{2} u=0
$$

The initial data is of Gaussian type

$$
u(x, 0)=\operatorname{sech}(x) \exp (2 \mathrm{i} x) .
$$

Here, $\gamma=1, \lambda=2$ are taken. Then, when $\alpha=1$, the soliton solution is

$$
u(x, t)=\operatorname{sech}(x-4 t) \exp \{\mathrm{i}(2 x-3 t)\} .
$$

Let $\Omega=(-20,20)$; we simulate the propagation of a single soliton with fixed $\tau=0.01$, $h=0.1$ and different $\alpha$. Fig. 1 displays the evolutions of the amplitudes of single solitons for $\alpha=0.6,0.8,0.9$, and 1, respectively, where the solitons of different width and height are observed. In particular, when $\alpha=1$, it collapses to the classical one. As depicted in [47], the order $\alpha(\alpha \neq 1)$ can create two turning points in the solitary wave solution; we see the same phenomena appear here from Fig. 2. Table 1 reports the convergent results at $t=1$ when $\alpha=1$ by selecting $\tau=0.1 * h$. Table 2 lists the values of conserved quantity $Q_{u}^{n}$ at 
Figure 2 The numerical solutions at different $t$ for $\alpha$ $=0.9$; red stars: $t=1$, blue circles: $t=2$, green triangles: $t=3$

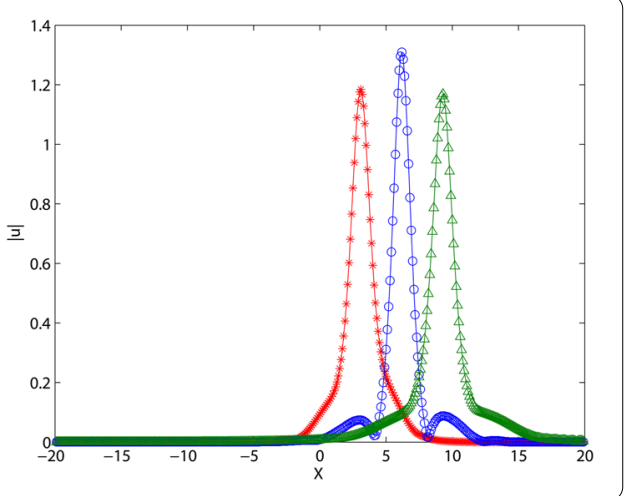

Table 1 The global errors and convergent orders at $t=1$ for $\alpha=1$ with $\tau=0.1 * h$

\begin{tabular}{llll}
\hline$\tau$ & $h$ & $\left\|u-u_{h}\right\|_{0, \Omega}$ & c. order \\
\hline 0.02 & 0.2 & $1.687612 \mathrm{e}-01$ & - \\
0.01 & 0.1 & $4.361017 \mathrm{e}-02$ & 1.952247 \\
0.005 & 0.05 & $1.101949 \mathrm{e}-02$ & 1.984607 \\
0.0025 & 0.025 & $2.765947 \mathrm{e}-03$ & 1.994212 \\
\hline
\end{tabular}

Table 2 The values of $Q_{u}^{n}$ at different $T$ for $\alpha=0.55,0.85$, and 1 with $\tau=h=0.05$

\begin{tabular}{llll}
\hline & $\alpha=0.55$ & $\alpha=0.85$ & $\alpha=1$ \\
\hline$T=0$ & 1.414213562373096 & 1.414213562373096 & 1.414213562373096 \\
$T=2$ & 1.414213563333915 & 1.414213562373732 & 1.414213562373095 \\
$T=4$ & 1.414213563483628 & 1.414213562459447 & 1.414213562373102 \\
$T=6$ & 1.414213565080403 & 1.414216796821000 & 1.414213562373097 \\
$T=8$ & 1.414213565803438 & 1.414334297978175 & 1.414213562373093 \\
\hline
\end{tabular}

different $T$ for $\alpha=0.55,0.85$, and 1. It is drawn that the mass is well preserved throughout the process and our scheme is available for long-time simulation.

Example 6.2 Consider the following space-fractional CNLS:

$$
\begin{aligned}
& \mathrm{i} \partial_{t} u+\gamma \partial_{x}^{2 \alpha} u+\lambda\left(|u|^{2}+\rho|v|^{2}\right) u=0, \\
& \mathrm{i} \partial_{t} v+\gamma \partial_{x}^{2 \alpha} v+\lambda\left(\rho|u|^{2}+|v|^{2}\right) v=0,
\end{aligned}
$$

subjected to the initial values

$$
\begin{aligned}
& u(x, 0)=\operatorname{sech}\left(x+x_{0}\right) \exp (\mathrm{i} c x), \\
& v(x, 0)=\operatorname{sech}\left(x-x_{0}\right) \exp (-\mathrm{i} c x) .
\end{aligned}
$$

As $\alpha=1$ and $\rho=1$, the model is known as the Manakov system, which is integrable, i.e., a collision without any reflection, transmission, trapping, and creation of new solitary waves would be produced, when two solitons intersect each other. Let $\gamma=1, x_{0}=5$, and $c=3$; we consider the double solitons collision and the impact may be caused by different fractional orders on the wave propagation within $\Omega=(-20,20)$ under the parameters $\tau=h=0.1$. In Fig. 3, we display the interaction of two solitons and the evolution of the amplitude of 

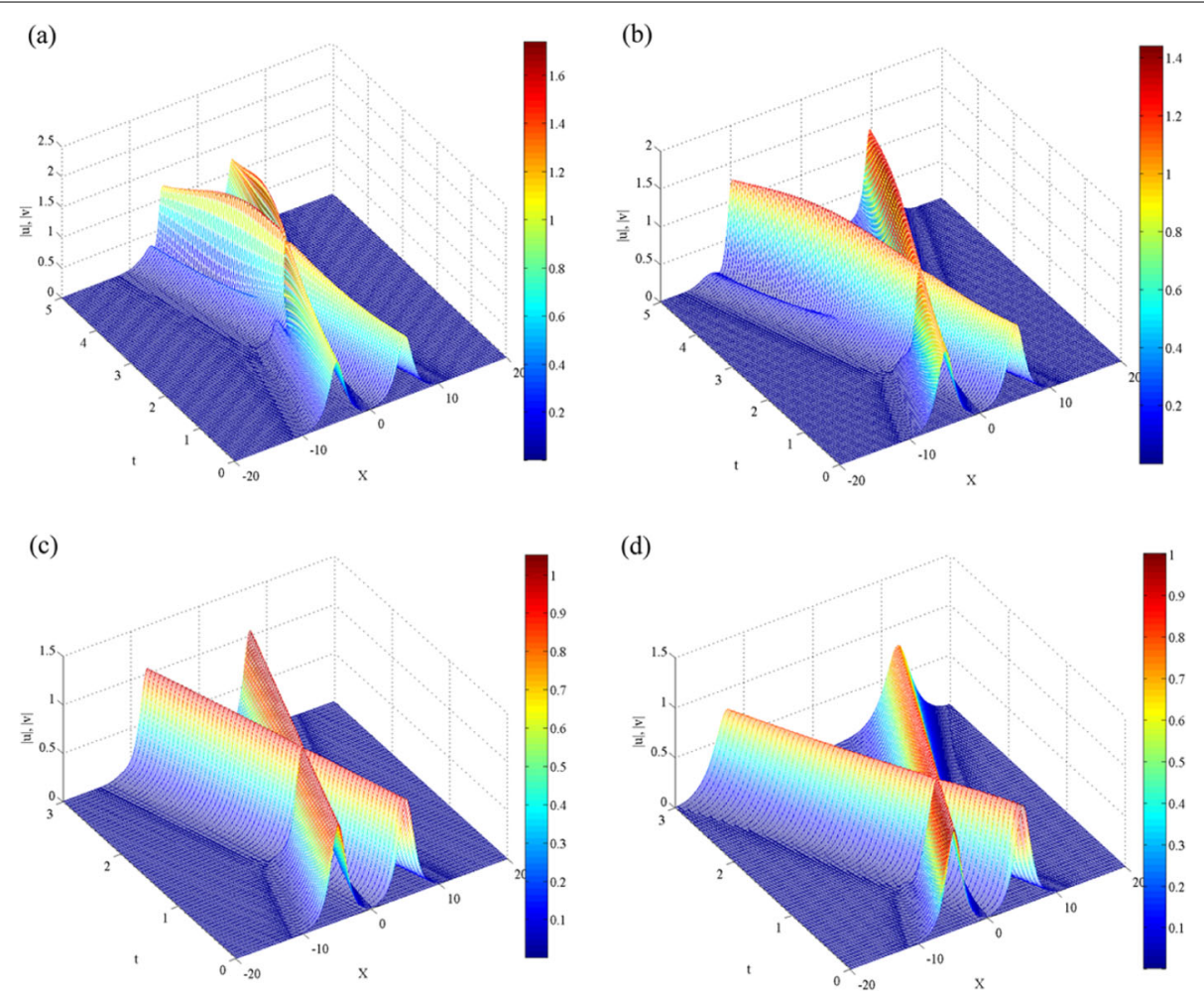

Figure 3 The interactions of two solitons for different $\alpha$ with $\lambda=\rho=1 ;(\mathbf{a}): \alpha=0.7,(\mathbf{b}): \alpha=0.8,(\mathbf{c}): \alpha=0.9$, (d): $\alpha=1$

each soliton when $\alpha=0.7,0.8,0.9$, and 1 . In Fig. 4, we present the state solutions at $t=1$ and $t=4$ for $\alpha=0.75$. It is observed that the width and height of the solitons have been greatly changed when $\alpha \neq 1$. More precisely, the smaller $\alpha$ we take, the bigger change will be created. The time point when the solitons intersect is also varying with $\alpha$. Besides, the shapes of the solitons may not be retained after they collide with each other when $\alpha \neq 1$. Table 3 reports the evolution of the quantity $Q_{u}^{n}$ for $\alpha=0.65,0.75$, and 1 , respectively, by which we easily see that our scheme well conserves the initial mass, where the quantity $Q_{v}^{n}$ is omitted since $Q_{v}^{n}=Q_{u}^{n}$.

At last, we compare the computing efficiency of the explicit-implicit FEM and the Crank-Nicolson FEM with Newton's iteration (termed "Newton Method"). Table 4 gives the detailed time costs for those two methods, from which we conclude that the explicitimplicit FEM is more efficient than the Newton Method.

Remarks 6.1 In the tests, Newton's procedure was terminated by reaching a solution with tolerant error 1.0E-12; all the algorithms were run by using Matlab R2012a on a Lenovo PC with Intel Pentium G2030 3.00 GHz CPU and 4 GB RAM.

\section{Conclusion}

In this research, a fully discrete mass conservative FEM is proposed to solve the Riesz space-fractional CNLS. Its convergence is discussed and the convergent orders are showed 
(a)

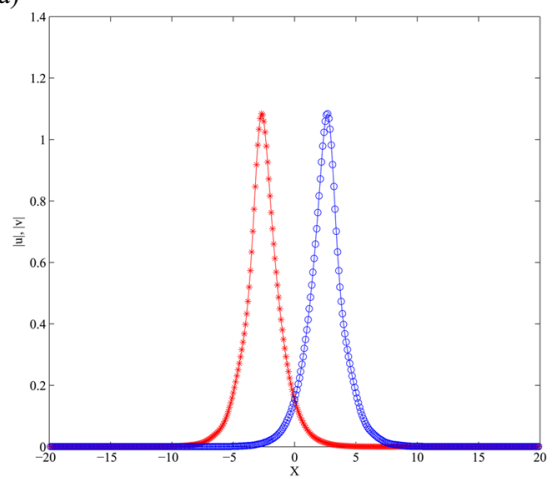

(b)

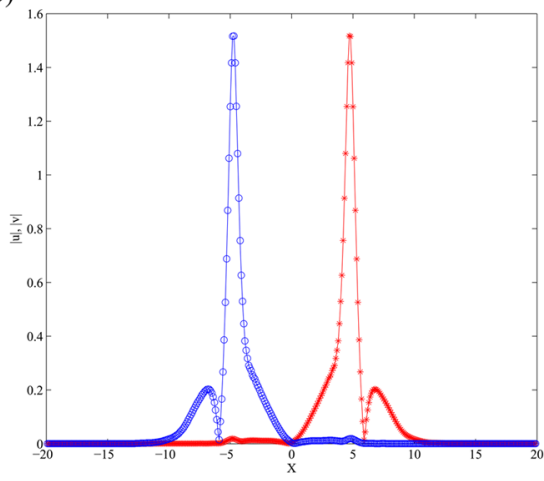

Figure 4 The numerical solutions at different $t$ for $\alpha=0.75 ;(\mathbf{a}): t=1,(\mathbf{b}): t=4$; red stars: $|u|$, blue circles: $|v|$

Table 3 The values of $Q_{u}^{n}$ at different $T$ for $\alpha=0.65,0.75$, and 1 with $\tau=h=0.1$

\begin{tabular}{llll}
\hline & $\alpha=0.65$ & $\alpha=0.75$ & $\alpha=1$ \\
\hline$T=0$ & 1.414213562373022 & 1.414213562373022 & 1.414213562373022 \\
$T=2$ & 1.414213562415025 & 1.414213562374943 & 1.414213562373022 \\
$T=4$ & 1.414213562434534 & 1.414213562393007 & 1.414213562373011 \\
$T=6$ & 1.414213563569567 & 1.414213562408873 & 1.414213562373014 \\
$T=8$ & 1.414213565171315 & 1.414213787517128 & 1.414213562373017 \\
\hline
\end{tabular}

Table 4 The time costs of Explicit-Implicit Method and Newton Method when $T=2$

\begin{tabular}{lllc}
\hline$\alpha$ & $\tau, h$ & Algorithm & CPU time (s) \\
\hline$\alpha=0.55$ & $0.04,0.1$ & Explicit-Implicit Method & 41.82 \\
& $0.04,0.1$ & Newton Method & 105.04 \\
$\alpha=0.75$ & $0.025,0.05$ & Explicit-Implicit Method & 504.05 \\
& $0.025,0.05$ & Newton Method & 977.68 \\
$\alpha=0.95$ & $0.02,0.03125$ & Explicit-Implicit Method & 2212.33 \\
& $0.02,0.03125$ & Newton Method & 5809.25 \\
\hline
\end{tabular}

to be optimal in $L^{2}$-sense. Since the scheme is linearized and decoupled, an extra Newton iterative loop is naturally avoided, which can significantly improve the computing efficiency in practice. The codes are also utilized to simulate the wave propagation of solitons, and a detailed numerical investigation on the double solitons collision is done with different fractional orders. The test results indicate the soliton shapes are greatly changed with $\alpha$, while the conserved quantities are well preserved. It is noteworthy that the problem discussed here can possibly be solved by other numerical methods like the lattice fractional equation methods in $[28,29]$, operational matrix methods in $[57,58]$, or the fourth-order nonstandard compact and sixth-order weighted essentially non-oscillatory (WENO) finite difference methods in $[59,60]$, which can reduce computing effort by converting the original problem to a system of differential/algebraic equations, or achieving high-order convergence without spurious oscillations. In future works, we will use the Riesz fractional differences and operational matrix techniques to deal with Riesz derivative or apply the ideas of the high-order nonstandard compact and WENO schemes in the above citations to derive more efficient numerical methods for Eqs. (1.1)-(1.2). Although some difficulties will be encountered, it is meaningful to extend these works to the space-fractional 
NLS/CNLS. The extensions of these methods to the CNLS with both time- and spacefractional derivatives will also be considered.

\author{
Acknowledgements \\ The authors are very grateful to the editor and anonymous referees for their kind help and valuable comments. \\ Funding \\ This research was supported by the National Natural Science Foundation of China (Nos.11471262 and 11601432).
}

Availability of data and materials

Not applicable.

Competing interests

The authors declare that they have no competing interests.

\title{
Authors' contributions
}

All authors contributed equally to this manuscript. All authors read and approved the final manuscript.

\section{Author details}

${ }^{1}$ School of Science, Shaoyang University, Shaoyang, P.R. China. ${ }^{2}$ Department of Applied Mathematics, Northwestern Polytechnical University, Xi'an, P.R. China.

\section{Publisher's Note}

Springer Nature remains neutral with regard to jurisdictional claims in published maps and institutional affiliations.

Received: 9 April 2019 Accepted: 31 July 2019 Published online: 08 August 2019

\section{References}

1. Yu, Q., Liu, F., Turner, I., Burrage, K., Vegh, V.: The use of a Riesz fractional differential-based approach for texture enhancement in image processing. ANZIAM J. 54, 590-607 (2013)

2. Baleanu, D., Jajarmi, A., Asad, J.H.: Classical and fractional aspects of two coupled pendulums. Rom. Rep. Phys. 71, 103 (2019)

3. Baleanu, D., Sajjadi, S.S., Jajarmi, A., Asad, J.H.: New features of the fractional Euler-Lagrange equations for a physical system within non-singular derivative operator. Eur. Phys. J. Plus 134, 181 (2019)

4. Mohammadi, F., Moradi, L., Baleanu, D., Jajarmi, A.: A hybrid functions numerical scheme for fractional optimal control problems: application to nonanalytic dynamic systems. J. Vib. Control 24(21), 5030-5043 (2018)

5. Hajipour, M., Jajarmi, A., Baleanu, D., Sun, H.G.: On an accurate discretization of a variable-order fractional reaction-diffusion equation. Commun. Nonlinear Sci. Numer. Simul. 69, 119-133 (2019)

6. Gorenflo, R., Mainardi, F., Moretti, D., Pagnini, G., Paradisi, P.: Discrete random walk models for space-time fractional diffusion. Chem. Phys. 284(1-2), 521-541 (2002)

7. Adams, E.E., Gelhar, L.W.: Field study of dispersion in a heterogeneous aquifer: 2. Spatial moments analysis. Water Resour. Res. 28(12), 3293-3307 (1992)

8. Nigmatulin, R.: The realization of the generalized transfer equation in a medium with fractal geometry. Phys. Status Solidi B 133, 425-430 (1986)

9. Barkai, E.: CTRW pathways to the fractional diffusion equation. Chem. Phys. 284, 13-27 (2002)

10. Gorenflo, R., Mainardi, F.: Random walk models for space-fractional diffusion processes. Fract. Calc. Appl. Anal. 1, 167-191 (1998)

11. Li, X.J., Xu, C.J.: Existence and uniqueness of the weak solution of the space-time fractional diffusion equation and a spectral method approximation. Commun. Comput. Phys. 8(5), 1016-1051 (2010)

12. Meerschaert, M.M., Scheffler, H.P., Tadjeran, C.: Finite difference methods for two-dimensional fractional dispersion equation. J. Comput. Phys. 211(1), 249-261 (2006)

13. Meerschaert, M.M., Tadjeran, C.: Finite difference approximations for fractional advection-dispersion flow equations. J. Comput. Appl. Math. 172(1), 65-77 (2004)

14. Sousa, E.: Finite difference approximations for a fractional advection diffusion problem. J. Comput. Phys. 228, 4038-4054 (2009)

15. Yuste, S.B.: Weighted average finite difference methods for fractional diffusion equations. J. Comput. Phys. 216, 264-274 (2006)

16. Zhuang, P., Liu, F.: Implicit difference approximation for the two-dimensional space-time fractional diffusion equation J. Appl. Math. Comput. 25, 269-282 (2007)

17. Deng, W.H.: Finite element method for the space and time fractional Fokker-Planck equation. SIAM J. Numer. Anal. 47, 204-226 (2008)

18. Ervin, V.J., Roop, J.P.: Variational formulation for the stationary fractional advection dispersion equation. Numer. Methods Partial Differ. Equ. 22, 558-576 (2006)

19. Zhang, H., Liu, F., Anh, V.: Galerkin finite element approximations of symmetric space fractional partial differential equations. Appl. Math. Comput. 217, 2534-2545 (2010)

20. Zhu, X.G., Nie, Y.F., Wang, J.G., Yuan, Z.B.: A numerical approach for the Riesz space-fractional Fisher' equation in two-dimensions. Int. J. Comput. Math. 94(2), 296-315 (2017)

21. Elsaid, A.: The variational iteration method for solving Riesz fractional partial differential equations. Comput. Math. Appl. 60, 1940-1947 (2010) 
22. Jafari, H., Tajadodi, H., Baleanu, D.: A modified variational iteration method for solving fractional Riccati differential equation by Adomian polynomials. Fract. Calc. Appl. Anal. 16, 109-122 (2013)

23. Zhu, X.G., Nie, Y.F., Zhang, W.W.: An efficient differential quadrature method for fractional advection-diffusion equation. Nonlinear Dyn. 90(3), 1807-1827 (2017)

24. Zhu, X.G., Yuan, Z.B., Liu, F.W., Nie, Y.F.: Differential quadrature method for space-fractional diffusion equations on 2D irregular domains. Numer. Algorithms 79(3), 853-877 (2018)

25. Sun, Z.Z., Wu, X.N.: A fully discrete difference scheme for a diffusion-wave system. Appl. Numer. Math. 56(2), 193-209 (2006)

26. Podlubny, I.: Fractional Differential Equations. Academic Press, San Diego (1999)

27. Ortigueira, M.D.: Riesz potential operators and inverses via fractional centred derivatives. Int. J. Math. Math. Sci. 2006, $48391(2006)$

28. Wu, G.-C., Baleanu, D., Deng, Z.-G., Zeng, S.-D.: Lattice fractional diffusion equation in terms of a Riesz-Caputo difference. Physica A 438, 335-339 (2015)

29. Wu, G.-C., Baleanu, D., Xie, H.-P.: Riesz Riemann-Liouville difference on discrete domains. Chaos, Interdiscip. J. Nonlinear Sci. 26, 084308 (2016)

30. Secchi, S.: Ground state solutions for nonlinear fractional Schrödinger equations in $\mathbb{R}^{N}$. J. Math. Phys. 54, 031501 (2013)

31. Benney, D.J., Newell, A.C.: The propagation of nonlinear wave envelops. J. Math. Phys. 46, 133-139 (1967)

32. Laskin, N.: Fractional quantum mechanics. Phys. Rev. E 62, 3135-3145 (2000)

33. Laskin, N.: Fractional quantum mechanics and Lévy path integrals. Phys. Lett. A 268, 298-305 (2000)

34. Guo, B.L., Han, Y.Q., Xin, J.: Existence of the global smooth solution to the period boundary value problem of fractional nonlinear Schrödinger equation. Appl. Math. Comput. 204, 468-477 (2008)

35. Guo, B.L., Huo, Z.H.: Well-posedness for the nonlinear fractional Schrödinger equation and inviscid limit behavior of solution for the fractional Ginzburg-Landau equation. Fract. Calc. Appl. Anal. 16, 226-242 (2013)

36. Hu, J.Q., Xin, J., Lu, H.: The global solution for a class of systems of fractional nonlinear Schrödinger equations with periodic boundary condition. Comput. Math. Appl. 62, 1510-1521 (2011)

37. Hong, Y., Sire, Y.: On fractional Schrödinger equations in Sobolev spaces. Commun. Pure Appl. Anal. 14(6), 2265-2282 (2015)

38. Chen, M.: The attractor of the dissipative coupled fractional Schrödinger equations. Math. Methods Appl. Sci. 37(5), 645-656 (2014)

39. Fall, M.M., Mahmoudi, F., Valdinoci, E.: Ground states and concentration phenomena for the fractional Schrödinger equation. Nonlinearity 28(6), 1937-1961 (2015)

40. Felmer, P., Quaas, A., Tan, J.G.: Positive solutions of the nonlinear Schrödinger equation with the fractional Laplacian. Proc. R. Soc. Edinb. A 142A, 1237-1262 (2012)

41. Hu, Y., Kallianpur, G.: Schrödinger equations with fractional Laplacians. Appl. Math. Optim. 42, 281-290 (2000)

42. Herzallah, M.A.E., Gepreel, K.A.: Approximate solution to the time-space fractional cubic nonlinear Schrödinger equation. Appl. Math. Model. 36, 5678-5685 (2012)

43. Atangana, A., Cloot, A.H.: Stability and convergence of the space fractional variable-order Schrödinger equation. Adv. Differ. Equ. 2013, 80 (2013)

44. Amore, P., Fernández, F.M., Hofmann, C.P., Sáenz, R.A.: Collocation method for fractional quantum mechanics. J. Math. Phys. 51, 122101 (2010)

45. Wang, P.D., Huang, C.M.: An energy conservative difference scheme for the nonlinear fractional Schrödinger equations. J. Comput. Phys. 293, 238-251 (2015)

46. Klein, C., Sparber, C., Markowich, P.: Numerical study of fractional nonlinear Schrödinger equations. Proc. R. Soc. A 470, $20140364(2014)$

47. Wang, D.L., Xiao, A.G., Yang, W.: Crank-Nicolson difference scheme for the coupled nonlinear Schrödinger equations with the Riesz space fractional derivative. J. Comput. Phys. 242, 670-681 (2013)

48. Wang, D.L., Xiao, A.G., Yang, W.: Maximum-norm error analysis of a difference scheme for the space fractional CNLS. Appl. Math. Comput. 257, 241-251 (2015)

49. Liu, Q., Zeng, F.H., Li, C.P.: Finite difference method for time-space-fractional Schrödinger equation. Int. J. Comput. Math. 92, 1439-1451 (2015)

50. Zhao, X., Sun, Z.Z., Hao, Z.P.: A fourth-order compact ADI scheme for two-dimensional nonlinear space fractional Schrödinger equation. SIAM J. Sci. Comput. 36, 2865-2886 (2014)

51. Huang, Y.Q., Li, X., Xiao, A.G.: Fourier pseudospectral method on generalized sparse grids for the space-fractional Schrödinger equation. Comput. Math. Appl. 75(12), 4241-4255 (2018)

52. Zhang, H., Jiang, X.Y., Wang, C., Fan, P.W.: Galerkin-Legendre spectral schemes for nonlinear space fractional Schrödinger equation. Numer. Algorithms 79(1), 337-356 (2017)

53. Zhu, X.G., Yuan, Z.B., Wang, J.G., Nie, Y.F., Yang, Z.Z.: Finite element method for time-space-fractional Schrödinger equation. Electron. J. Differ. Equ. 2017, 166 (2017)

54. Li, M., Gu, X.M., Huang, C.M., Fei, M.F., Zhang, G.Y.: A fast linearized conservative finite element method for the strongly coupled nonlinear fractional Schrödinger equations. J. Comput. Phys. 358, 256-282 (2018)

55. Bu, W.P., Liu, X.T., Tang, Y.F., Yang, J.Y.: Finite element multigrid method for multi-term time fractional advection diffusion equations. Int. J. Model. Simul. Sci. Comput. 6, 1540001 (2015)

56. Li, B.Y., Sun, W.W.: Unconditional convergence and optimal error estimates of a Galerkin-mixed FEM for incompressible miscible flow in porous media. SIAM J. Numer. Anal. 51(4), 1959-1977 (2013)

57. Sadeghi Roshan, S., Jafari, H., Baleanu, D.: Solving FDEs with Caputo-Fabrizio derivative by operational matrix based on Genocchi polynomials. Math. Methods Appl. Sci. 41(18), 9134-9141 (2018)

58. Baleanu, D., Shiri, B., Srivastava, H.M., Al Qurashi, M.: A Chebyshev spectral method based on operational matrix for fractional differential equations involving non-singular Mittag-Leffler kernel. Adv. Differ. Equ. 2018, 353 (2018)

59. Hajipour, M., Jajarmi, A., Baleanu, D.: On the accurate discretization of a highly nonlinear boundary value problem. Numer. Algorithms 79(3), 679-695 (2018)

60. Hajipour, M. Jajarmi, A., Malek, A., Baleanu, D.: Positivity-preserving sixth-order implicit finite difference weighted essentially non-oscillatory scheme for the nonlinear heat equation. Appl. Math. Comput. 325, 146-158 (2018) 